\title{
Information encoded in volumes and areas of dendritic spines is nearly maximal across mammalian brains
}

\author{
Jan Karbowski ${ }^{1, *}$, Paulina Urban ${ }^{2,3,4}$ \\ ${ }^{1}$ Institute of Applied Mathematics and Mechanics, University of Warsaw, Warsaw, Poland; \\ ${ }^{2}$ Laboratory of Functional and Structural Genomics, Centre of New Technologies, University of \\ Warsaw, Warsaw, Poland; ${ }^{3}$ College of Inter-Faculty Individual Studies in Mathematics and Natural \\ Sciences, University of Warsaw, Warsaw, Poland; ${ }^{4}$ Laboratory of Databases and Business Analytics, \\ National Information Processing Institute, National Research Institute, Warsaw, Poland.
}

\begin{abstract}
Long-term information associated with neuronal memory resides in dendritic spines. However, spines can have a limited size due to metabolic and neuroanatomical constraints, which should effectively limit the amount of encoded information in excitatory synapses. This study investigates how much information can be stored in the sizes of dendritic spines, and whether is it optimal in any sense? It is shown here, using empirical data for several mammalian brains across different regions and physiological conditions, that dendritic spines nearly maximize entropy contained in their volumes and surface areas for a given mean size. This result is essentially independent of the type of a fitting distribution to size data, as both short- and heavy-tailed distributions yield similar nearly $100 \%$ information efficiency in the majority of cases, although heavy-tailed distributions slightly better fit the data. On average, the highest information is contained in spine volume, and the lowest in spine length or spine head diameter. Depending on a species and brain region, a typical spine can encode between 6.1 and 10.8 bits of information in its volume, and 3.1-8.1 bits in its surface area. Our results suggest a universality of entropy maximization in spine volumes and areas, which can be a new principle of memory storing in synapses.
\end{abstract}

Keywords: Dendritic spine size distributions, Information encoded in spines; Synaptic memory efficiency; Entropy maximization; Constraints.

* Corresponding author: jkarbowski@mimuw.edu.pl 


\section{Significance statement}

It is believed that memory in the brain is stored in the parts of excitatory synapses called dendritic spines. But how efficient is the memory capacity given synaptic size variability? Generally, the larger the synapse the more information can be packed in its structure. However, this process comes at some cost, as larger synapses use more metabolic energy and brain tissue. Thus, from a theoretical point of view, there exists a benefit-cost trade-off for storing long-term information in dendritic spines, with a unique optimal solution set by an average spine size. We show that volumes and areas of actual spines in different parts of the brain across different mammals are very close to that optimal theoretical solution for storing information. This means that synaptic information is maximally optimized regardless of brain size, region, and physiological condition, which is a remarkable result. 


\section{INTRODUCTION}

Dendritic spines in excitatory neurons are the storing sites of long-term memory, mainly in their molecular components, i.e., receptors and proteins (Kennedy 2000; Kasai et al 2003; Chklovskii et al 2004; Lisman et al 2012; Huganir and Nicoll 2013; Meyer et al 2014; Takeuchi et al 2014). Experimental data show that spines vary vastly in sizes and shapes (Bonhoeffer and Yuste 2002; Holtmaat et al 2005; Yasumatsu et al 2008), but their size highly correlates with a magnitude of synaptic current (synaptic weight or strength), which suggests that there is a close relationship between spine structure and physiological function (Kasai et al 2003). Moreover, the variability in spine's size is also strictly associated with a variability in the number of AMPA receptors on spine membrane (Nusser et al 1998), as well as with changeability in the size of postsynaptic density (PSD) (Harris et al 1992), which is composed of the thousands of proteins implicated in molecular learning and memory storage (Lisman et al 2012; Sheng and Hoogenraad 2007).

The tight correlations between spine geometry and spine molecular composition, responsible for encoding and maintaining of memory, suggest that one can use the spine size as some measure of its information content. On the other hand, despite spine size variability, its mean value should be restricted due to limitations of cortical space associated with dense packing of different neuronal and non-neuronal components (Chklovskii et al 2002; Karbowski 2015). Moreover, because of the linear relationship between average spine size and postsynaptic current (Kasai et al 2003), bigger spines with stronger synaptic strength are generally more energy consuming than smaller spines with weaker strength (Attwell and Laughlin 2001; Karbowski 2012 and 2021; Laughlin et al 1998). These considerations suggest that molecular information encoded in the geometry of dendritic spines must be limited both by neuroanatomical and metabolic constraints, in particular, by the average spine size.

The main goal of this study is to investigate the information capacity of dendritic spines contained 
in the distribution of their sizes. The specific questions we ask are the following: Is such information optimized in some sense, given the constraints on spine sizes? If so, how large is the deviation from the optimality for the parameters characterizing spine distributions? To answer these questions, we collected data from published literature on spine (or PSD) sizes (volumes, areas, length, and head width) for divers mammals and different cortical and subcortical regions (see the Methods). These data allowed us to compute empirical Shannon entropy associated with spine sizes for species, brain region and condition, and to compare it with a theoretical upper bound on the entropy for a given mean spine size. 


\section{RESULTS}

Fitting of dendritic spine sizes to lognormal, loglogistic, and gamma distributions, and empirical Shannon entropy.

Previous empirical studies on dendritic spines have shown that their sizes (or synaptic weights) can be fitted well to either lognormal or gamma distributions (Benavides-Piccione et al 2013; Loewenstein et al 2011; Song et al 2005; Santuy et al 2020; Tamada et al 2020). However, these two types of the probability distributions differ significantly in terms of their asymptotic behavior: for very large spine sizes the former displays a heavy tail, while the latter decays exponentially with a short tail. Our first goal is to determine which distribution, with heavy or short tail, can better describe experimental data. In our analysis, we also added an additional probability density, not tried by previous studies, the loglogistic distribution that has a heavy tail. This distribution has an interesting property, because for small sizes it behaves similar to the gamma distribution, whereas for large sizes it resembles the lognormal distribution. For this reason, the loglogistic function is an intermediate alternative to the two extreme choices used in the past (Benavides-Piccione et al 2013; Loewenstein et al 2011; Song et al 2005; Santuy et al 2020; Tamada et al 2020).

In Figs. 1 and 2 we present fits of the empirical spine volume and length from human cingulate cortex, taken from Benavides-Piccione et al (2013), to the three mentioned theoretical distributions. The best fits for spine volume are loglogistic probability densities, both for 40 and 85 years old individuals (Kolmogorov-Smirnov test: loglogistic statistic yields $0.48-0.53$ vs. $0.50-0.56$ for gamma statistic, and $0.95-0.96$ for lognormal). For spine length the best fit for both individuals is lognormal distribution (KS test: lognormal statistic yields $0.26-0.51$ vs. 0.94 for gamma and loglogistic). Note that the best fits in all cases are the probability densities with heavy tails.

Next, we want to determine how stable are the distributions of spine sizes across the whole develop- 
mental period (Figs. 3 and 4). To address this, we use another collection of data on spine length and spine head diameter from human hippocampus (Das et al 2019; and private comm.). It is important to emphasize that spine length histograms seem visually invariant from the early age of 2 years to 71 years old, with the exception of 5 month old for which the histogram is broader though with essentially the same mean (Fig. 3). This qualitative observation is supported by statistics: all the spine length data can be described best by lognormal distribution (KS test statistic: $0.50-0.51$ for lognormal vs. $0.83-0.86$ for loglogistic and $0.85-0.88$ for gamma). Interestingly, this is also the same distribution that best fits the other data on spine length in cingulate cortex present in Fig. 2. The case for spine head diameter is slightly more complex, despite a visual similarity of all data histograms throughout the whole development (Fig. 4). For this parameter, the fitting distributions can alternate between the three probability densities (Fig. 4). For the youngest individual (5 months old) the best fit yields loglogistic function (KS statistic 0.37), for two older individuals (70 and 71 years old) the best fit is provided by lognormal (KS statistic 0.50 ), while for 8 intermediate individuals (from 2 to 68 years old) the best fit gives gamma distribution (KS statistic $0.39-0.48)$.

Our next goal is to find an empirical Shannon entropy associated with the discrete histograms of spine sizes in Figs. 1-4, and to compare it to continuous entropies associated with the three fitting probability distributions. This is conducted using Eq. 1 in the Methods for the continuous fitting distributions, and using a discrete version of this equation for the histograms. Calculations reveal that the continuous distributions provide entropies that very well approximate the empirical entropies for all fits in Figs. 1-4, with small differences between the two mostly no more than $3-4 \%$. For example, for the data in Figs. 1 and 2, the empirical entropies associated with spine volume yield 9.55 bits for 40 years old and 9.66 bits for 85 years old, while the continuous entropies give 9.39 (lognormal), 9.22 (loglogistic), 9.57 (gamma) for 40 years old, and 9.51 (lognormal), 9.35 (loglogistic), 9.69 (gamma) 
for 85 years old. For spine length empirical entropy we obtain 4.75 bits for 40 years old and 4.64 for 85 years old, while for the continuous entropies we get 4.70 (lognormal), 4.60 (loglogistic), 4.80 (gamma) for 40 years old, and 4.73 (lognormal), 4.62 (loglogistic), 4.83 (gamma) for 85 years old. Similar agreement is also visible for the data in Figs. 3 and 4. For spine length empirical entropy in human hippocampus we have, e.g.: 4.47 bits (5 months), 4.58 bits (27 years), and 4.55 bits (71 years old), which should be compared with the continuous entropies: 4.59 (lognormal), 4.48 (loglogistic), 4.71 (gamma) for 5 months old; 4.56 (lognormal), 4.46 (loglogistic), 4.67 (gamma) for 27 years old; and 4.52 (lognormal), 4.41 (loglogistic), 4.63 (gamma) for 71 years old. For spine head diameter empirical entropy in human hippocampus we have, e.g.: 2.68 bits (5 months), 2.76 bits (27 years), and 2.81 bits (71 years old), whereas for the continuous entropies we obtain: 2.58 (lognormal), 2.49 (loglogistic), 2.68 (gamma) for 5 months old; 2.69 (lognormal), 2.59 (loglogistic), 2.79 (gamma) for 27 years old; and 2.71 (lognormal), 2.61 (loglogistic), 2.82 (gamma) for 71 years old.

To summarize, our fitting analysis reveals that the empirical data on spine sizes can be described well by different continuous distributions, but in most cases the best fits are obtained for the densities with heavy tails (either lognormal or loglogistic). Furthermore, the entropies associated with these distributions give reasonably good approximations to the empirical entropies.

\section{Information contained in spine sizes is nearly maximal for spine volume and area.}

We collected a large data set of dendritic spine sizes from different sources (Tables 1-4; and Methods). These data contain values of mean and standard deviations of spine (or PSD) volume, surface area, length, and spine head diameter in different brain regions of several mammalian species. Next, we make an assumption, based on the results in Figs. 1-4, that all the collected data on spine sizes can be described well by the three discussed above distributions, and additionally that the entropies associated with these continuous distributions are good approximations of the empirical entropies 
related to empirical, mostly unknown, distributions of the collected data in Tables 1-4. With this logic in mind, we are able to estimate the entropy associated with each spine size parameter for the three distributions (Eq. 1 in the Methods), using only the means and standard deviations of the data. We call the values of these entropies as empirical entropies $\left(H_{g}, H_{l n}, H_{l l}\right.$; Eqs. 5, 10, 16), or equivalently the empirical information content in dendritic spines. Additionally, we compute a theoretical upper bound on the entropy for a given mean spine size for each distribution $\left(H_{g, m}, H_{l n, m}, H_{l l, m} ;\right.$ Eqs. 8, 13, 21). By comparing empirical entropies $H$ to their upper bounds $H_{m}$, we can assess how close to the theoretical optimum is the information contained in spine sizes, which is quantified by an efficiency of information $\eta$ defined as the ratio of $H$ and $H_{m}$ (Eq. 22 in the Methods).

Another relevant quantity that we compute is deviation from the optimality $D\left(D_{g}, D_{l n}, D_{l l}\right)$, which measures a deviation of the two parameters characterizing a given distribution from their optimal values defining a maximal entropy (Eqs. 23-25 in the Methods). This quantity is analogous to a standard error, which means its smallness is an indicator of the closeness of the empirical parameters to their optimal values.

The results in Figs. 5 and 6 for spine volume and area (including PSD volumes and areas) indicate that the corresponding empirical entropies essentially reach their upper theoretical bounds. This fact is also evident from a nearly $100 \%$ of the information efficiency ratio $\eta$, as well as from a relatively small values of the deviation from optimality $D$ (Tables 1 and 2 ). This is a remarkable result showing that regardless of brain size, brain region, age, or neurophysiological condition, the distributions of spine volume and area adjust themselves such that to maximize their information content. The maximal values of the entropy depend logarithmically on the mean spine volume or area, and are in the range 3-10 bits per spine, depending on average spine size. Generally, the distributions with heavy tails (lognormal, loglogistic) are slightly closer to the theoretical optimum than the short-tailed gamma 
distribution.

The results for spine length and spine head diameter show that the corresponding empirical entropies are more distant to the theoretical optimum than those for spine volume or area (lower values of the efficiency $\eta$; Tables 3 and 4). Nevertheless, the spine length entropies, although not at the vicinity of the upper bound, are significantly closer to it than spine head diameter entropies (Figs. 7 and 8; Tables 3 and 4). The latter deviate considerably, as can be seen from the large values of $D$ (Table 4).

Taken together, the results shown in Figs. 5-8 indicate that more information is encoded in spine volume and area than spine length or diameter. Moreover, the information associated with volume and surface area is maximal possible for given mean values of volume and area.

\section{Density of information in spines is not optimal.}

As an alternative to the problem of information maximization in dendritic spines, we consider also the possibility that not entropy but the entropy density could be maximized. In particular, we study the ratio $F$ of empirical entropy to mean spine size (volume, area, length, and diameter), as a relevant quantity for maximization (Eq. 26 in the Methods). From a theoretical point of view, for each of the three considered distributions the entropy density $F$ exhibits a single maximum. The height of that maximum corresponds to the upper bound on the entropy density, and our goal is to investigate how much the empirical $F$ differs from that bound.

In Fig. 9 we present the empirical values of the entropy density and compare them to the maximal values of $F$. These results show that the empirical densities of entropy are generally far lower than their upper bounds. Specifically, the empirical entropy density $F$ is only $\sim 1-10 \%$ or $\sim 10-50 \%$ of its maximal values for spine volume and area, respectively. The empirical ratio $F$ for spine length and head diameter are higher, but still far away from the upper bound, at $\sim 30-70 \%$ of the maximal $F$.

We conclude that the density of entropy is not optimized in dendritic spines, which suggests that 
bioRxiv preprint doi: https://doi.org/10.1101/2021.12.30.474505; this version posted December 30, 2021. The copyright holder for this

preprint (which was not certified by peer review) is the author/funder, who has granted bioRxiv a license to display the preprint in perpetuity. It is made available under aCC-BY-NC-ND 4.0 International license.

this quantity is not the most relevant for information encoding in synapses. 


\section{Discussion}

Summary of the main results: optimization of information encoded in spine volume and area, but not in spine length or head diameter.

Our results suggest that the sizes of dendritic spines can be fitted equally well by three different skewed distributions. However, the distributions with heavy tails generally slightly better describe the empirical data (see Figs. 1-4), especially spine volume and length.

The main result of this study is that information encoded in spine volume and area is essentially optimized, achieving in many cases a nearly $100 \%$ of its upper bound value for a given mean size of spine volume or area (Tables 1 and 2; Figs. 5 and 6). This conclusion is also supported by relatively small deviations from the optimality of the parameters characterizing the distributions $(D$ values mostly below $20-30 \%$ for heavy-tailed distributions). Furthermore, the optimality of information in spine volume or area is independent of the investigated species (i.e. brain size), brain region (except cerebellum), age, or condition (Tables 1 and 2), which might suggest its possible universality, at least in the cerebral cortex. The exception of the cerebellum, with only $75-77 \%$ of its information efficiency is intriguing, but from a theoretical point of view it is a consequence of the uniformity of spine sizes (though not of PSD sizes) in that part of the brain (Harris and Stevens 1988).

In most cases, the optimality of information in spine volumes and areas is also independent of the type of the chosen spine size distribution. The few exceptions in Tables 1 and 2 reflect the fact that the gamma distribution poorly handles cases in which standard deviation exceeds the mean. That is also the reason for several negative entropy values associated with this distribution in Table 1, which is only a mathematical artifact.

The efficiency of the information encoded in spine length and spine head (or PSD) diameter is significantly lower than that encoded in spine volume and area (compare Tables 3 and 4 with Tables 
1 and 2). Despite the relatively high efficiency values $\eta$ for spine length (around $80-90 \%$ in many cases), the deviations from optimality $D$ are generally much larger than for spine volume or area. For the spine head diameter the information efficiency $\eta$ is generally the lowest and more scattered, mostly in the range $50-90 \%$, and the deviations from optimality are even higher than for spine length (especially for gamma distribution). Taken together, these results suggest that neither spine length nor spine head diameter maintain the synaptic information optimally.

It is also shown here that the density of information is not optimized in any way, as its empirical values are far below their upper bounds (Fig. 9). This indicates that entropy density is not the relevant quantity for neuroanatomical and functional organization of dendritic spines, if we take an evolutionary point of view into account (Parker and Maynard-Smith 1990; Striedter 2005).

Finally, there have been a few studies that are similar in spirit to our general approach but that differ in context and details. Notably, Brunel et al (2004) and Varshney et al (2004) assumed an information maximization under constraints to derive optimal distribution of synaptic weights and to make some general qualitative observations. In contrast, we took a different approach and we actually proved the maximization of information in synaptic volumes and areas in different parts of the brain across several mammals. This was achieved using the collected data and fitting procedures for different distributions of synaptic sizes.

\section{Stability of information contained in spine sizes and memory.}

The aim of this study was to provide answers to two basic questions associated with the relationship between dendritic spines and memory. The first is: in which synaptic structural parameter information is most likely encoded? The second question is about the optimality of the encoded information. Both of the questions are interrelated.

We found that a typical dendritic spine can store $6.1-10.8$ bits in its volumes, and $3.1-8.1$ bits 
in its surface area (Tables 1 and 2). Our number for spine volume is $1.5-2$ times greater than the amount of information determined for spine head volume in rat hippocampus by Bartol et al (2015). The discrepancy can be attributed to a different method of information estimation by these authors. We computed Shannon information directly from the empirical distributions, while Bartol et al (2015) used a signal detection theory and an assumption of partial Gaussian distributions of sample points, which might not be an accurate estimate. Our results indicate that spines in the mammalian cerebral cortex can hold about $(3-5) \cdot 10^{12} \mathrm{bits} / \mathrm{cm}^{3}$ of memory, assuming in agreement with the data that cortical synaptic density is roughly brain size independent and on average $5 \cdot 10^{11} \mathrm{~cm}^{-3}$ (Karbowski 2014; Sherwood et al 2020).

Although synaptic information can in principle be stored in many different structural synaptic parameters, it seems that the most relevant are those parameters that allow the maximal possible storage. This is what one can expect based on the principle of evolutionary pressure for optimizing the relevant variables (Parker and Maynard-Smith 1990; Striedter 2005). In this sense, and considering our results, the most likely parameter in which memory can be encoded is spine volume or area, and these parameters can serve as useful empirical indicators of a precise information content in individual synapses. Moreover, given that excitatory postsynaptic current and the number of AMPA receptors ("synaptic weight") are proportional to spine volume (Kasai et al 2003), this conclusion is consistent with a general hypothesis held in neuroscience that memory is encoded in excitatory synaptic weights.

Why memory can be more efficiently encoded in spine volume or area than e.g. spine length or diameter. It seems that volume offers more "states", as it intrinsically contains more degrees of freedom than any other linear size. Additionally, the fact that spine volume and area are equally good for storing information can be a consequence of their tight correlations (Bartol et al 2015). On the other hand, very weak correlations between spine length and spine volume (Bartol et al 2015; Tamada 
et al 2020) can explain the suboptimality of information storage in spine length (Fig. 7).

It is also curious that information efficiency $\eta$ is relatively stable even in the cases when the condition changes. For example, for situations stress vs. non-stress, or mutation vs. control, or LTP vs. nonLTP condition, the information efficiency stays remarkable constant despite changes in the mean and standard deviations of spine sizes (Tables 1-4). This stability might suggest that some compensatory mechanisms take plays in synapses that counteract their memory degradation. Interestingly, the same conclusion can be reached for the developmental data on human hippocampus (Das et al 2019), where the corresponding information encoded in spine length and head diameter is remarkably invariant across the human lifespan (Tables 3 and 4 ).

Implications for neuroanatomical and metabolic organization of the cerebral cortex in the context of synaptic information capacity.

It is known from physics that information is always associated with energy (Leff and Rex 1990). There have been suggestions that information processing in neurons is energy efficient (Laughlin et al 1998; Levy and Baxter 1996; Levy and Calvert 2021; Rieke et al 1999). We have an analogous situation in this study for dendritic spines: Long-term information contained in spine volumes and areas is maximized for a given mean spine size, which is directly proportional to the spine energy consumption during synaptic transmission (Attwell and Laughlin 2001; Karbowski 2012; Laughlin et al 1998). In this light, our result is essentially an additional example of the energetic efficiency of information, this time on a synaptic level and on a long time scale.

The nearly optimal encoding of information in dendritic spine volume and area, found in this study, could be a new principle of neuroanatomical and functional organization of synaptic system in the mammalian cerebral cortex. Maximization of stored information in these synaptic parameters is independent of the cortical region and physiological conditions, and this suggests an universality 
of optimal encoding in synapses. In contrast, numerical spine density along dendrites generally lacks cross-regional uniformity, as it is greater in prefrontal than in somatosensory or visual cortical areas (Bianchi et al 2013; Elston et al 2006). Consequently, it seems that spine information content is decoupled from a linear spine dendritic density.

Another prominent principle, indirectly related, is associated with optimization (minimization) of neuronal wiring length or volume (Chklovskii et al 2002; Kaiser and Hilgetag 2006; Karbowski 2001; Mitchison 1991). It was shown that minimization of axonal and dendritic processes leads to an efficient use of metabolic energy in the brain (Attwell and Laughlin 2001; Chklovskii et al 2002), especially when the brain is scaled up in the evolutionary process (Herculano-Houzel 2011; Karbowski 2007; Yu et al 2014). A recent, interesting result shows that the entropy associated with the length of neuronal wiring is maximized (Song et al 2021). This result is complementary to ours, and both results suggest an efficient encoding of long-term information in the whole neuron. This conclusion is in line with the observation of optimal design of dendritic arbors for information content maximization (Wen et al 2009).

An alternative to neural wiring optimization, or its extension, is the principle of spine economy, which can explain the hierarchical organization of different components in the mammalian cerebral cortex (Karbowski 2015). It seems that it would be interesting to explore a connection of the spine economy principle with our results on the optimization of information in dendritic spines. It might lead to some new theoretical insight on the links between neuroanatomy and information content in cortical circuits, e.g. (Shepherd et al 2005; Bourne and Harris 2008; Hill et al 2012). 


\section{METHODS}

\section{Data collection and analysis for the sizes of dendritic spines.}

All the experimental data on the sizes of dendritic spines used in this study were collected from different published sources, and concern several mammals. Data for mouse brain come from: (Arellano et al 2007; Benavides-Piccione et al 2002; Ishii et al 2018; Kashiwagi et al 2019; Konur et al 2003; Parajuli et al 2020; Rodriguez-Moreno et al 2018; Santuy et al 2020; Scheuss and Bonhoeffer 2014; Schikorski and Steven 1999; Tamada et al 2020), for rat brain from (Borczyk et al 2019; Cheetham et al 2014; Harris and Stevens 1988, 1989; Radley et al 2008; Swulius et al 2010), for rabbit brain from (Schwartzkroin and Kunkel 1982), for echidna brain from (Hassiotis et al 2003), for cat brain from (Clemo et al 2016; da Costa 2013), for macaque monkey brain from (Lubke et al 2015; Medalla and Barbas 2009; Medalla and Luebke 2015; Motley et al 2018; Young et al 2014), for dolphin brain from (Glezer and Morgane 1990), and for human brain from (Alonso-Nanclares et al 2008; BenavidesPiccione et al 2002; Benavides-Piccione et al 2013; Boros et al 2019; Das et al 2019; Tang et al 2001). The data used are both from cortical and subcortical regions, at different animal ages and different physiological conditions. The cortical areas include: piriform, somatosensory, visual, prefrontal, cingulate, parietal, temporal, and auditory cortex. The subcortical regions include: hippocampus, striatum, cerebellum, and forebrain.

The data for fitting distributions of dendritic spine sizes were taken from Benavides-Piccione et al (2013) (human cingulate cortex) and from Das et al (2019) (human hippocampus). These data were fitted to the three distributions (gamma, lognormal, and loglogistic) using standard tools from Matlab. We used the Kolmogorov-Smirnov test as a measure of the goodness of each fit (James 2006). 


\section{Theoretical modeling.}

\section{Maximization of entropy.}

Shannon Entropy or information content $H$, contained in the probability distribution $\rho(x)$ of some random variable $x(0 \leq x \leq \infty)$ is defined as (Dayan and Abbott 2001)

$$
H=-\frac{1}{\ln (2)} \int_{0}^{\infty} d x \rho(x) \ln [\rho(x) \Delta x]
$$

where $\Delta x$ is the resolution of the variable $x$ (its smallest empirically detectable value). Below, we consider $x$ to be either spine volume, spine surface area (or PSD area), spine head diameter, or spine length. Consequently, the parameter $\Delta x$ is different for volume, area, and linear size. In accordance with the principles of spatial resolution used in light microscopy (on which the data we use are based), we choose $\Delta x=0.1 \mu \mathrm{m}$ for linear dimensions, $\Delta x=0.01 \mu \mathrm{m}$ for areas, and $\Delta x=0.001 \mu \mathrm{m}$ for volumes, e.g. (Kashiwagi et al 2019). Our numerical results for entropy are to a large extent insensitive on the precise values of $\Delta x$, because of the logarithmic dependence of the entropy $H$ on $\Delta x$ (Eq. 1). Thus changing $\Delta x$ by a factor of as much as 3 , leads only to a marginal change in the calculated entropy.

In what follows, we solve the following optimization problem: we want to maximize the entropy given a fixed mean value of $x$. Keeping fixed the mean value of $x$ reflects the constraint coming from neuroanatomical and/or metabolical restrictions on spine size. Mathematically, this procedure is equivalent to the standard Lagrange optimization problem with the Lagrangian $L$ defined as

$$
L=H-\lambda(\langle x\rangle-S)
$$


where $\langle x\rangle$ is the mean (average) of $x$ over the distribution $\rho(x)$, the parameter $S$ is the average dendritic spine size (either volume, area, head diameter, or length), and $\lambda$ is the Lagrange multiplier. The resulting maximal entropy will be a function of spine size $S$ (see below).

\section{Entropy of dendritic spines with gamma distribution.}

The gamma distribution of a random variable $x$ (spine size) is skewed but it decays fast for large values of $x$. It is defined as

$$
\rho_{g}(x)=\frac{\beta^{\alpha}}{\Gamma(\alpha)} x^{\alpha-1} e^{-\beta x}
$$

where $\rho_{g}(x)$ is the probability density of $x$, the parameters $\alpha$ and $\beta$ are some positive numbers, and $\Gamma(\alpha)$ is the Gamma function.

The entropy for the gamma distribution $H_{g}$ is found from Eqs. (1) and (3), which generates

$$
H_{g}(\alpha, \beta)=-\frac{1}{\ln (2)}\left[\ln \left(\frac{\beta^{\alpha} \Delta x}{\Gamma(\alpha)}\right)-\beta\langle x\rangle_{g}+(\alpha-1) \int_{0}^{\infty} d x \rho_{g}(x) \ln (x)\right]
$$

The integral on the right hand side can be evaluated explicitly with the help of the formula (Gradshteyn and Ryzhik 2007)

$$
\int_{0}^{\infty} d x x^{\alpha-1} e^{-\beta x} \ln (x)=\Gamma(\alpha)[\psi(\alpha)-\ln (\beta)] / \beta^{\alpha},
$$

where $\psi(\alpha)$ is the Digamma function (Gradshteyn and Ryzhik 2007). Additionally, the average of $x$ for this distribution is $\langle x\rangle_{g}=\alpha / \beta$. Combining these results we obtain the entropy $H_{g}$ as 


$$
H_{g}(\alpha, \beta)=\frac{1}{\ln (2)}\left[\alpha+(1-\alpha) \psi(\alpha)+\ln \left(\frac{\Gamma(\alpha)}{\beta \Delta x}\right)\right]
$$

The standard deviation for the gamma distribution (defined as $\sigma_{g}=\sqrt{\left\langle x^{2}\right\rangle_{g}-\langle x\rangle_{g}^{2}}$ ) is $\sigma_{g}=\sqrt{\alpha} / \beta$. We can invert the relations for the mean and standard deviations to find the parameters $\alpha$ and $\beta$ for given experimental values of $\langle x\rangle_{g}$ and $\sigma_{g}$. The result is:

$$
\begin{array}{r}
\alpha=\left(\frac{\langle x\rangle_{g}}{\sigma_{g}}\right)^{2} \\
\beta=\frac{\langle x\rangle_{g}}{\sigma_{g}^{2}} .
\end{array}
$$

Note that the entropy $H_{g}(\alpha, \beta)$ can become negative for $\alpha \ll 1$, since in this limit the Digamma function behaves as $\psi(\alpha) \approx-1 / \alpha$. This situation corresponds to the cases in which $\sigma_{g} \gg\langle x\rangle_{g}$, i.e. to the data points for which standard deviation is much greater than the mean.

We find the maximal entropy of the gamma distribution given a fixed $\langle x\rangle_{g}$ by solving the Lagrange optimization problem defined in Eq. (2). The optimal parameters $\alpha$ and $\beta$ are found by setting $\partial L / \partial \alpha=0, \partial L / \partial \beta=0$, and $\partial L / \partial \lambda=0$. As a result, their optimal values are $\alpha_{0}=1, \beta_{0}=1 / S$, and $\lambda_{0}=-1 / S$. For this values, the maximal entropy of the gamma distribution is:

$$
H_{g, m}=\ln \left(\frac{e S}{\Delta x}\right) / \ln (2)
$$

where $e$ is the Euler number $(e \approx 2.71)$. Interestingly, $H_{g, m}$ is also the maximal possible entropy given a fixed mean for all distributions of $x$. 


\section{Entropy of dendritic spines with lognormal distribution.}

The lognormal distribution of a random variable $x$ is both skewed and has a heavy tail, and is defined

as

$$
\rho_{l n}(x)=\frac{1}{\sqrt{2 \pi} \sigma x} \exp \left[-\frac{(\ln x-\mu)^{2}}{2 \sigma^{2}}\right],
$$

where $\rho_{l n}(x)$ is the probability density of $x$, and $\mu, \sigma$ are some parameters $(\sigma>0)$.

The entropy of the lognormal distribution $H_{l n}$ can be determined by combining Eqs. (1) and (9). Using the substitution $y=\ln (x)$ the corresponding integrals can be evaluated similarly as in the case of gaussian distribution, which is a standard procedure. As a result, the entropy of lognormal distribution takes the form

$$
H_{l n}(\mu, \sigma)=\frac{1}{\ln (2)}\left[\frac{1}{2}+\mu+\ln \left(\frac{\sqrt{2 \pi} \sigma}{\Delta x}\right)\right]
$$

The average of $x$ for this distribution is $\langle x\rangle_{l n}=\exp \left(\mu+\sigma^{2} / 2\right)$, and the standard deviation $\left(\sigma_{\ln }=\right.$ $\left.\sqrt{\left\langle x^{2}\right\rangle_{l n}-\langle x\rangle_{l n}^{2}}\right)$ is $\sigma_{l n}=\langle x\rangle_{l n} \sqrt{e^{\sigma^{2}-1}}$. By inverting these relations, we find

$$
\begin{array}{r}
\mu=\ln \left(\frac{\langle x\rangle_{l n}^{2}}{\sqrt{\langle x\rangle_{l n}^{2}+\sigma_{l n}^{2}}}\right) \\
\sigma=\sqrt{\ln \left(1+\left(\sigma_{l n} /\langle x\rangle_{l n}\right)^{2}\right)} .
\end{array}
$$


Equations (11-12) allow us to find the characteristic parameters $\mu$ and $\sigma$ defining the lognormal distribution from the experimental values of mean and standard deviation for the variable $x$.

As before, we find the maximal entropy of the lognormal distribution given a fixed $\langle x\rangle_{l n}$ by solving the Lagrange optimization problem defined in Eq. (2). The optimal parameters $\mu$ and $\sigma$ are found by setting $\partial L / \partial \mu=0, \partial L / \partial \sigma=0$, and $\partial L / \partial \lambda=0$. Their optimal values are: $\mu_{0}=-0.5+\ln (S), \sigma_{0}=1$, and $\lambda_{0}=-1 / S$. For this values, the maximal entropy of the lognormal distribution is:

$$
H_{l n, m}=\ln \left(\frac{\sqrt{2 \pi} S}{\Delta x}\right) / \ln (2),
$$

and it is clear that $H_{l n, m}<H_{g, m}$.

\section{Entropy of dendritic spines with loglogistic distribution.}

The loglogistic distribution of a random variable $x$ is visually similar to the lognormal distribution with heavy tail, except that it decays as power law for very large $x$. The loglogistic probability density $\rho_{l l}$ is defined as

$$
\rho_{l l}(x)=\frac{b}{a} \frac{(x / a)^{b-1}}{\left[1+(x / a)^{b}\right]^{2}}
$$

where $a$ and $b$ are some positive parameters. Note that for $x \gg a$ the probability density $\rho_{l l}$ behaves asymptotically as $\rho(x)_{l l} \sim 1 / x^{b+1}$, which is a much slower decay than for the gamma distribution.

The entropy of the loglogistic distribution $H_{l l}$ can be determined by combining Eqs. (1) and (14). Consequently, we have 


$$
H_{l l}(a, b)=\frac{1}{\ln (2)}\left[\ln \left(\frac{a}{b \Delta x}\right)-(b-1) \int_{0}^{\infty} d x \rho_{l l}(x) \ln \left(\frac{x}{a}\right)+2 \int_{0}^{\infty} d x \rho_{l l}(x) \ln \left(1+\left(\frac{x}{a}\right)^{b}\right)\right]
$$

The first integral on the right hand side (without the prefactor $(b-1)$ ) is performed by the substitution $y=(x / a)^{b}$. This transforms that integral into

$$
(1 / b) \int_{0}^{\infty} d y \frac{\ln (y)}{(1+y)^{2}}
$$

which is equal to 0 (Gradshteyn and Ryzhik 2007). The second integral on the right hand side (without the prefactor 2) can be transformed, using the same substitution, into

$$
\int_{0}^{\infty} d y \frac{\ln (1+y)}{(1+y)^{2}}
$$

which has a value equal to 1 (Gradshteyn and Ryzhik 2007). As a result, the entropy of loglogistic distribution takes the form

$$
H_{l l}(a, b)=\frac{1}{\ln (2)}\left[2+\ln \left(\frac{a}{b \Delta x}\right)\right]
$$

The mean $\langle x\rangle_{l l}$ and standard deviation $\sigma_{l l}$ for this distribution both exist, i.e. they are finite, provided the parameter $b>2$. In this case, we have

$$
\begin{array}{r}
\langle x\rangle_{l l}=\frac{\pi a / b}{\sin (\pi / b)} \\
\sigma_{l l}=a \sqrt{\frac{2 \pi / b}{\sin (2 \pi / b)}-\frac{(\pi / b)^{2}}{\sin ^{2}(\pi / b)}} .
\end{array}
$$


The inverse relations between the parameters $a, b$ and $\langle x\rangle_{l l}, \sigma_{l l}$ are given by

$$
\begin{aligned}
& \tan (\pi / b)=\left(1+\frac{\sigma_{l l}^{2}}{\langle x\rangle_{l l}^{2}}\right)\left(\frac{\pi}{b}\right) \\
& a=\langle x\rangle_{l l}\left(1+\frac{\sigma_{l l}^{2}}{\langle x\rangle_{l l}^{2}}\right) \cos (\pi b) .
\end{aligned}
$$

The first equation has to be solved numerically, for given empirical values of $\langle x\rangle_{l l}, \sigma_{l l}$. The second equation determines $a$ once the parameter $b$ is known.

The maximal value of the entropy for loglogistic distribution given a fixed $\langle x\rangle_{l l}$ is found by solving the Lagrange optimization problem defined in Eq. (2). The optimal parameters $a$ and $b$ are found by setting $\partial L / \partial a=0, \partial L / \partial b=0$, and $\partial L / \partial \lambda=0$. Their optimal values are $a_{0}=2 S / \pi, b_{0}=2$, and $\lambda_{0}=-1 / S$. For this values, the maximal entropy of the loglogistic distribution is:

$$
H_{l l, m}=\ln \left(\frac{e^{2} S}{\pi \Delta x}\right) / \ln (2),
$$

and we also have that $H_{l l, m}<H_{g, m}$, as expected.

\section{Definition of information efficiency.}

Information efficiency $\eta$ is defined as the ratio of the empirical entropy $\left(H_{l n}, H_{l l}, H_{g}\right)$ to the theoretical upper bound of entropy for a given spine size $\left(H_{l n, m}, H_{l l, m}, H_{g, m}\right)$. Thus, for a specific probability distribution we have 


$$
\eta_{\alpha}=\frac{H_{\alpha}}{H_{\alpha, m}}
$$

where $\alpha$ denotes one of the distributions (either $l n, l l$, or $g$ ).

\section{Deviation from optimality of the empirical parameters characterizing spine size distribu-}

\section{tions.}

We introduce a measure of deviation of the parameters characterizing a given distribution from their optimal theoretical values, as a relative combined error from optimality.

For the gamma distribution the deviation $D_{g}$ is:

$$
D_{g}=\frac{1}{\sqrt{2}} \sqrt{\left(\frac{\alpha-\alpha_{0}}{\alpha_{0}}\right)^{2}+\left(\frac{\beta-\beta_{0}}{\beta_{0}}\right)^{2}} .
$$

For the log-normal distribution the deviation $D_{l n}$ is:

$$
D_{l n}=\frac{1}{\sqrt{2}} \sqrt{\left(\frac{\mu-\mu_{0}}{\mu_{0}}\right)^{2}+\left(\frac{\sigma-\sigma_{0}}{\sigma_{0}}\right)^{2}},
$$

and for the log-logistic distribution the deviation $D_{l l}$ is:

$$
D_{l l}=\frac{1}{\sqrt{2}} \sqrt{\left(\frac{a-a_{0}}{a_{0}}\right)^{2}+\left(\frac{b-b_{0}}{b_{0}}\right)^{2}} .
$$

In Eqs. (23-25) the parameters $\alpha_{0}, \beta_{0}, \mu_{0}, \sigma_{0}$, and $a_{0}, b_{0}$ are the optimal parameters for a given distribution. The smaller the value of $D$, the closer the empirical distribution is to its maximal entropy.

\section{Alternative measure of optimality: Maximization of entropy density.}

As an alternative to the above approach with the maximization of the entropy for a given average 
spine size, we consider below the maximization of the density of entropy. More precisely, we analyze the optimization problem in which we want to maximize the entropy of spine sizes per average spine size.

The fitness function $F$ in this case takes the form:

$$
F=\frac{H}{\langle x\rangle}
$$

where $\langle x\rangle$ is the mean spine size as above. Interestingly, the function $F$ displays maxima for each of the three probability densities.

For the gamma distribution, we have the following form of the fitness function $F_{g}(\alpha, \beta)=H_{g} /\langle x\rangle_{g}$ :

$$
F_{g}(\alpha, \beta)=\frac{\beta}{\alpha \ln (2)}\left[\alpha+(1-\alpha) \psi(\alpha)+\ln \left(\frac{\Gamma(\alpha)}{\beta \Delta x}\right)\right]
$$

Optimal values of $\alpha$ and $\beta$ are found from the conditions: $\partial F_{g}(\alpha, \beta) / \partial \alpha=0$, and $\partial F_{g}(\alpha, \beta) / \partial \beta=0$. A straightforward calculation leads to the following optimal values: $\alpha_{0}=1$, and $\beta_{0}=1 / \Delta x$, for which the maximal value of fitness function $F_{g, m} \equiv F_{g}\left(\alpha_{0}, \beta_{0}\right)$ is

$$
F_{g, m}=\frac{1}{\Delta x \ln (2)}
$$

For the lognormal distribution, we have the following form of the fitness function $F_{l n}(\mu, \sigma)=$ $H_{l n} /\langle x\rangle_{l n}:$ 


$$
F_{l n}(\mu, \sigma)=\frac{1}{\ln (2)}\left[\frac{1}{2}+\ln \left(\frac{\sqrt{2 \pi} \sigma}{\Delta x}\right)+\mu\right] e^{-\left(\mu+\sigma^{2} / 2\right)}
$$

The optimal values $\mu_{0}, \sigma_{0}$ of $\mu$ and $\sigma$ are found from the conditions: $\partial F_{l n}(\mu, \sigma) / \partial \mu=0$, and $\partial F_{l n}(\mu, \sigma) / \partial \sigma=$

0. This procedure yields: $\mu_{0}=1 / 2+\ln (\Delta x / \sqrt{2 \pi})$ and $\sigma_{0}=1$, and the corresponding maximal value of entropy density $F_{l n, m}=F_{l n}\left(\mu_{0}, \sigma_{0}\right)$, which is

$$
F_{l n, m}=\frac{\sqrt{2 \pi}}{e \Delta x \ln (2)}
$$

For the loglogistic distribution, we have the following form of the fitness function $F_{l l}(a, b)=$ $H_{l l} /\langle x\rangle_{l l}$ :

$$
F_{l l}(a, b)=\frac{b}{\pi a \ln (2)} \sin \left(\frac{\pi}{b}\right) \ln \left(\frac{e^{2} a}{b \Delta x}\right)
$$

As before maximization of $F_{l l}(a, b)$ is performed by solving the following conditions: $\partial F_{l l}(a, b) / \partial a=0$, and $\partial F_{l l}(a, b) / \partial b=0$. This procedure yields the optimal parameters of $a$ and $b: a_{0}=2 \Delta x / e$ and $b_{0}=2$, and the maximal value of $F_{l l}$ as

$$
F_{l l, m}=\frac{e}{\pi \Delta x \ln (2)}
$$




\section{Acknowledgments}

We would like to thank Dr. Ruth Benavides-Piccione and Dr. Sujan Chandra Das for providing us the datasets of their published results on dendritic spine sizes in humans. The work was supported by the Polish National Science Centre (NCN) grant number 2021/41/B/ST3/04300 (JK).

\section{Competing interests}

There is no competing interests to declare. 


\section{References}

Alonso-Nanclares L, Gonzalez-Sorlano J, Rodriguez JR, DeFelipe J (2008) Gender differences in human cortical synaptic density. Proc. Natl. Acad. Sci. USA 105: 14615-14619.

Arellano JI, Benavides-Piccione R, DeFelipe J, Yuste R (2007) Ultrastructure of dendritic spines: correlation between synaptic and spine morphologies. Front. Neurosci. 1: 131-143.

Attwell D, Laughlin SB (2001) An energy budget for signaling in the gray matter of the brain. $J$. Cereb. Blood Flow Metabol. 21: 1133-1145.

Bartol TM, Bromer C, Kinney J, Chirillo MA, Bourne JN, Harris KM, Sejnowski TJ (2015) Nanoconnectomic upper bound on the variability of synaptic plasticity. eLife 4: e10778.

Benavides-Piccione R, Ballesteros-Yanez I, DeFelipe J, Yuste R (2002) Cortical area and species differences in dendritic spine morphology. J. Neurocytol. 31: 337-346.

Benavides-Piccione R, Fernaud-Espinosa I, Robles V, Yuste R, DeFelipe J (2013) Age-based comparison of human dendritic spine structure using complete three-dimensional reconstructions. Cerebral Cortex 23: 1798-1810.

Bianchi S, Stimpson CD, Bauernfeind AL, Schapiro SJ, Baze WB, et al (2013) Dendritic morphology of pyramidal neurons in the chimpanzee neocortex: Regional specializations and comparison to humans. Cereb. Cortex 23: 2429-2436.

Bonhoeffer T, Yuste R (2002) Spine motility: phenomenology, mechanisms, and function. Neuron 35: 1019-1027.

Borczyk M, Sliwinska MA, Caly A, Bernas T, Radwanska K (2019) Neuronal plasticity affects correlation between the size of dendritic spine and its postsynaptic density. Scientific Rep. 9: 1693.

Boros BD, Greathouse KM, Gearing M, Herskowitz JH (2019) Dendritic spine remodeling accompanies Alzheimer's disease pathology and genetic susceptibility in cognitively normal aging. Neurobiology of 
Aging 73: 92-103.

Bourne JN, Harris KM (2008) Balancing structure and function at hippocampal dendritic spines. Annual Rev. Neurosci. 31: 47-67.

Brunel N, Hakim V, Isope P, Nadal JP, Barbour B (2004) Optimal information storage and the distribution of synaptic weights: perceptron versus Purkinje cell. Neuron 43: 745-757.

Cheetham CEJ, Barnes SJ, Albieri G, Knott GW, Finnert GT (2014) Pansynaptic enlargement at adult cortical connections strengthened by experience. Cereb. Cortex 24: 521-531.

Chklovskii DB, Mel BW, Svoboda K (2004) Cortical rewiring and information storage. Nature 431: $782-788$.

Chklovskii DB, Schikorski T, Stevens CF (2002) Wiring optimization in cortical circuits. Neuron 34: $341-347$.

Clemo HR, Lomber SG, Meredith MA (2016) Synaptic basis for cross-modal plasticity: enhanced supragranual dendritic spine density in anterior ectosylvian auditory cortex of the early deaf cat. Cereb. Cortex 26: $1365-1376$.

da Costa NM (2013) Diversity of thalamorecipient spine morphology in cat visual cortex and its implication for synaptic plasticity. J. Comp. Neurol. 521: 2058-2066.

Das SC, Chen D, Callor WB, Christensen E, Coon H, Williams ME (2019) Dil-mediated analysis of presynaptic and postsynaptic structures in human postmortem brain tissue. J. Comp. Neurol. 527: 3087-3098.

Dayan P, Abbott LF (2001) Theoretical Neuroscience. Cambridge, MA: MIT Press.

Elston GN, Benavides-Piccione R, Elston A, Zietsch B, DeFelipe J, et al (2006) Specializations of the granular prefrontal cortex of primates: Implications for cognitive processing. Anatomical Record A 288A: 26-35. 
Glezer II, Morgane PJ (1990) Ultrastructure of synapses and golgi analysis of neurons in neocortex of the lateral gyrus (visual cortex) of the dolphin and pilot whale. Brain Res. Bulletin 24: 401-427.

Gradshteyn IS, Ryzhik IM (2007) Table of Integrals, Series, and Products. Elsevier: Amsterdam (17th edit.).

Harris KM, Stevens JK (1988) Dendrritic spines of rat cereberal purkinje cells: serial electron microscopy with reference to their biophysical characteristics. J. Neurosci. 8: 4455-4469.

Harris KM, Stevens JK (1989) Dendrritic spines of CA1 pyramidal cells in the rat hippocampus: serial electron microscopy with reference to their biophysical characteristics. J. Neurosci. 9: 2982-2997.

Harris KM et al (1992) Three-dimensional structure of dendritic spines and synapses in rat hippocampus (CA1) at postnatal day 15 and adult ages: implications for the maturation of synaptic physiology and long-term potentiation. J. Neurosci. 12: 2685-2705.

Hassiotis M, Paxinos G, Ashwell KWS (2003) The anatomy of the cerebral cortex of the echidna (Tachyglossus aculeatus). Comp. Biochem. Physiol. A 136: 827-850.

Herculano-Houzel S (2011) Scaling of brain metabolism with a fixed energy budget per neuron: Implications for neuronal activity, plasticity and evolution. PLoS ONE 6: e17514.

Hill SL, Wang Y, Riachi I, Schurmann F, Markram H (2012) Statistical connectivity provides a sufficient foundation for specific functional connectivity in neocortical neural microcircuits. Proc. Natl. Acad. Sci. USA 109: E2885-E2894.

Holtmaat AJ, Trachtenberg JT, Wilbrecht L, Shepherd GM, Zhang X, et al (2005) Transient and persistent dendritic spines in the neocortex in vivo. Neuron 45: 279-291.

Huganir RL, Nicoll RA (2013) AMPARs and synaptic plasticity: the last 25 years. Neuron 80: 704717.

Ishii K, Nagaoka A, Kishida Y, Okazaki H, Yagishita S, Ucar H, Takahashi N, Saito N, Kasai H 
(2018). In vivo dynamics of dendritic spines in the neocortex of wild-type and Fmr1 KO mice. eNeuro

\section{5: ENEURO.0282-18.2018.}

James F (2006) Statistical Methods in Experimental Physics. London: World Scientific, 2 edition.

Kaiser M, Hilgetag CC (2006) Nonoptimal component placement, but short processing paths, due to long-distance projections in neural systems. PLoS Comput. Biol. 2: e95.

Karbowski J (2001) Optimal wiring principle and plateaus in the degree of separation for cortical neurons. Phys. Rev. Lett. 86: 3674-3677.

Karbowski J (2007) Global and regional brain metabolic scaling and its functional consequences. $B M C$ Biology 5: 18.

Karbowski J (2012) Approximate invariance of metabolic energy per synapse during development in mammalian brains. PLoS ONE 7: e33425.

Karbowski J (2014) Constancy and trade-offs in the neuroanatomical and metabolic design of the cerebral cortex. Front. Neural Circuits 8: 9.

Karbowski J (2015) Cortical composition hierarchy driven by spine proportion economical maximization or wire volume minimization. PloS Comput. Biol. 11: e1004532.

Karbowski J (2021) Energetics of stochastic BCM type synaptic plasticity and storing of accurate information. J. Comput. Neurosci. 49: 71-106.

Kasai H, Matsuzaki M, Noguchi J, Yasumatsu N, Nakahara H (2003) Structure-stability-function relationships of dendritic spines. Trends Neurosci. 26: 360-368.

Kashiwagi Y, Higashi T, Obashi K, Sato Y, Komiyama NH, Grant SGN, Okabe S (2019) Computational geometry analysis of dendritic spines by structured illumination microscopy. Nature Comm. 10: 1285.

Kennedy MB (2000) Signal-processing machines at the postsynaptic density. Science 290: 750-754. 
Konur S, Rabinowitz D, Fenstermaker V, Yuste R (2003) Systematic regulation of spine head diameters and densities in pyramidal neurons from juvenile mice. J. Neurobiol. 56: 95-112.

Laughlin SB, de Ruyter van Steveninck RR, Anderson JC (1998) The metabolic cost of neural information. Nature Neurosci. 1: 36-40.

Leff HS, Rex AF (1990) Maxwell's Demon: Entropy, Information, Computing. Princeton, NJ: Princeton Univ. Press.

Levy WB, Baxter RA (1996) Energy efficient neural codes. Neural Computation 8: 531-543.

Levy WB, Calvert VG (2021) Communication consumes 35 times more energy than computation in the human cortex, but both costs are needed to predict synapse number. Proc. Natl. Acad. Sci. USA 118: e2008173118.

Lisman J, Yasuda R, Raghavachari S (2012) Mechanisms of CaMKII action in long-term potentiation. Nat. Rev. Neurosci. 13: 169-182.

Loewenstein Y, Kuras A, Rumpel S (2011) Multiplicative dynamics underlie the emergence of the log-normal distribution of spine sizes in the neocortex in vivo. J. Neurosci. 31: 9481-9488.

Luebke JI, Medalla M, Amatrudo JM, Weaver CM, Crimins JL, Hunt B, Hof PR, Peters A (2015) Age-related changes to layer 3 pyramidal cells in the rhesus monkey visual cortex. Cereb. Cortex 25: 1454-1468.

Medalla M, Barbas H (2009) Synapses with inhibitory neurons differentiate anterior cingulate from dorsolateral prefrontal pathways associated with cognitive control. Neuron 61: 609-620.

Medalla M, Luebke JI (2015) Diversity of glutamatergic synaptic strength in lateral prefrontal versus primary visual cortices in the rhesus monkey. J. Neurosci. 35: 112-127.

Meyer D, Bonhoeffer T, Scheuss V (2014) Balance and stability of synaptic structures during synaptic plasticity. Neuron 82: 430-443. 
Mitchison G (1991) Neuronal branching patterns and the economy of cortical wiring. Proc. R. Soc. Lond. B 245: 151-158.

Motley SE, Grossman YS, Janssen WGM, Baxter MG, Rapp PR, Dumitriu D, Morrison JH (2018) Selective loss of thin spines in area $7 \mathrm{a}$ of the primate intraparietal sulcus predicts age-related working memory impairment. J. Neurosci. 38: 10467-10478.

Nusser Z et al (1998) Cell type and pathway dependence of synaptic AMPA receptor number and variability in the hippocampus. Neuron 21: 545-559.

Parajuli LK, Wako K, Maruo S, Kakuta S, Taguchi T, Ikuno M, Yamakado H, Takahashi R, Koike M (2020) Developmental changes in dendritic spine morphology in the striatum and their alteration in an A53T $\alpha$-synuclein transgenic mouse model of Parkinson's disease. eNeuro 7: ENEURO.0072-20.2020. Parker GA, Maynard Smith J (1990) Optimality theory in evolutionary biology. Nature 348: 27-33.

Radley JJ, Rocher AB, Rodriguez A, Ehlenberger DB, Dammann M, Mcewen BS, Morrison JH, Wearne SL, Hof PR (2008) Repeated stress alters dendritic spine morphology in the rat medial prefrontal cortex. J. Comp. Neurol. 507: 1141-1150.

Rieke F, Warland D, de Ruyter R, Bialek W (1999) Spikes: Exploring the neural code. Cambridge, MA: MIT Press.

Rodriguez-Moreno J, et al (2018) Quantitative 3D ultrastructure of thalamocortical synapses from the "lemniscal" ventral posteromedial nucleus in mouse barrel cortex. Cereb. Cortex 28: 3159-3175.

Santuy A, et al (2020) Estimation of the number of synapses in the hippocampus and brain-wide by volume electron microscopy and genetic labeling. Scientific Reports 10: 14014.

Scheuss V, Bonhoeffer T (2014) Function of dendritic spines on hippocampal inhibitory neurons. Cereb. Cortex 24: $3142-3153$.

Schikorski T, Stevens CF (1999) Quantitative fine-structural analysis of olfactory cortical synapses. 
Proc. Natl. Acad. Sci. USA 96: 4107-4112.

Schwartzkroin PA, Kunkel DD (1982) Electrophysiology and morphology of the developing hippocampus of fetal rabbits. J. Neurosci. 2: 448-462.

Sheng M, Hoogenraad CC (2007) The postsynaptic architecture of excitatory synapses: a more quantitative view. Annu. Rev. Biochem. 76: 823-847.

Shepherd GM, Stepanyants A, Bureau I, Chklovskii D, Svoboda K (2005) Geometric and functional organization of cortical circuits. Nat. Neurosci. 8: 782-790.

Sherwood CC, Miller SB, Karl M, Stimpson CD, Phillips KA, et al (2020) Invariant synapse density and neuronal connectivity scaling in primate neocortical evolution. Cereb. Cortex 30: 5604-5615.

Song S, Sjöström PJ, Reigl M, Nelson SB, Chklovskii DB (2005) Highly nonrandom features of synaptic connectivity in local cortical circuits. PLoS Biol. 3: e68.

Song Y, Zhou D, Li S (2021) Maximum entropy principle underlies wiring length distribution in brain networks. Cereb. Cortex 31: 4628-4641.

Striedter GF (2005) Principles of Brain Evolution. Sunderland, MA: Sinauer Assoc.

Swulius MT, Kubota Y, Forest A, Waxham MN (2010) Structure and composition of the postsynaptic density during development. J. Comp. Neurol. 518: 4243-4260.

Takeuchi T, Duszkiewicz AJ, Morris RGM (2014) The synaptic plasticity and memory hypothesis: encoding, storage and persistence. Phil. Trans. R. Soc. B 369: 20130288.

Tamada H, Blanc J, Korogod N, Petersen CCH, Knott GW (2020) Ultrastructural comparison of dendritic spine morphology preserved with cryo and chemical fixation. eLife $\mathbf{9}$ : e56384.

Tang Y, Nyengaard JR, de Groot DMG, Gundersen HJG (2001) Total regional and global number of synapses in the human brain neocortex. Synapse 41: 258-273.

Wen Q, Stepanyants A, Elston G, Grosberg AY, Chklovskii DB (2009) Maximization of the connec- 
tivity repertoire as a statistical principle governing the shapes of dendritic arbors. Proc. Natl. Acad. Sci. USA 106: 12536-12541.

Varshney LR, Sjostrom PJ, Chklovskii DB (2006) Optimal information storage in noisy synapses under resource constraint. Neuron 52: 409-423.

Yasumatsu N, Matsuzaki M, Miyazaki T, Noguchi J, Kasai H (2008) Principles of long-term dynamics of dendritic spines. J. Neurosci. 28: 13592-13608.

Young ME, Ohm DT, Dumitriu D, Rapp PR, Morrison JH (2014) Differential effects of aging on dendritic spines in visual cortex and prefrontal cortex of the rhesus monkey. Neuroscience 274: 33-43.

Yu YG, Karbowski J, Sachdev RNS, Feng JF (2014) Effect of temperature and glia in brain size enlargement and origin of allometric body-brain size scaling in vertebrates. BMC Evol. Biol. 14 : 178. 


\section{Figure Captions}

Fig. 1

Fitting of spine volume to lognormal, loglogistic, and gamma distributions. Empirical histograms of spine volumes from human cingulate cortex (rectangles; combined spines from apical and basal dendrites taken from Benavides-Piccione (2013)) were fitted to three different distributions (solid lines). A) Fits for 40 years old yield the following parameters: $\mu=-1.56, \sigma=0.87$ (lognormal); $a=0.22, b=2.03$ (loglogistic); and $\alpha=1.67, \beta=5.72$ (gamma). Goodness of fit using KS test: statistic $=0.963, p=7 \cdot 10^{-144}($ lognormal $) ;$ statistic $=0.533, p=4 \cdot 10^{-27}(\log \operatorname{logistic}) ;$ statistic $=0.561$, $p=3 \cdot 10^{-30}$ (gamma). B) Fits for 85 years old give: $\mu=-1.50, \sigma=0.91$ (lognormal); $a=0.24$, $b=1.93$ (loglogistic); and $\alpha=1.56, \beta=4.91$ (gamma). Goodness of fit using KS test: statistic $=0.949$, $p=2 \cdot 10^{-130}($ lognormal $) ;$ statistic $=0.482, p=6 \cdot 10^{-22}($ loglogistic $) ;$ statistic $=0.501, p=8 \cdot 10^{-24}$ (gamma).

Fig. 2

Fitting of spine length to lognormal, loglogistic, and gamma distributions. Empirical histograms of spine length from human cingulate cortex (rectangles; combined spines from apical and basal dendrites taken from Benavides-Piccione (2013)) were fitted to three different distributions (solid lines). A) Fits for 40 years old yield: $\mu=0.34, \sigma=0.52$ (lognormal); $a=1.46, b=3.44$ (loglogistic); and $\alpha=4.31, \beta=2.71$ (gamma). Goodness of fit using KS test: statistic $=0.505, p=3 \cdot 10^{-24}$ (lognormal); statistic $=0.944, p=1 \cdot 10^{-125}$ (loglogistic); statistic $=0.948, p=7 \cdot 10^{-129}$ (gamma). B) Fits for 85 years old yield: $\mu=0.33, \sigma=0.52$ (lognormal); $a=1.44, b=3.42$ (loglogistic); and $\alpha=4.26, \beta=2.70$ (gamma). Goodness of fit using KS test: statistic $=0.262, p=1.6 \cdot 10^{-6}$ (lognormal); statistic $=0.938$, $p=1 \cdot 10^{-121}(\log \operatorname{logistic}) ;$ statistic $=0.944, p=1 \cdot 10^{-125}$ (gamma). 
Fig. 3

Similarity of spine length distributions across human lifespan. Empirical data for human hippocampal spine length (rectangles; taken from Das et al (2019)) ranging from infancy (except 5 month old), through maturity, to senility look very similar. These data were fitted to three different distributions (solid lines). A) Fitting parameters for lognormal: $\mu=0.14, \sigma=0.59$, KS statistic $=0.508$, $p=2 \cdot 10^{-24}(5$ months $) ; \mu=0.17, \sigma=0.55, \mathrm{KS}$ statistic $=0.503, p=6 \cdot 10^{-24}(2$ years $) ; \mu=0.19$, $\sigma=0.52, \mathrm{KS}$ statistic $=0.502, p=8 \cdot 10^{-24}(23$ years $) ; \mu=0.20, \sigma=0.53, \mathrm{KS}$ statistic $=0.502$, $p=8 \cdot 10^{-24}(27$ years $) ; \mu=0.20, \sigma=0.52, \mathrm{KS}$ statistic $=0.502, p=8 \cdot 10^{-24}(38$ years $) ; \mu=0.18$, $\sigma=0.53, \mathrm{KS}$ statistic $=0.501, p=9 \cdot 10^{-24}(45$ years $) ; \mu=0.17, \sigma=0.52, \mathrm{KS}$ statistic $=0.501$, $p=9 \cdot 10^{-24}(57$ years $) ; \mu=0.16, \sigma=0.53, \mathrm{KS}$ statistic $=0.501, p=9 \cdot 10^{-24}(58$ years $) ; \mu=0.16$ $\sigma=0.53, \mathrm{KS}$ statistic $=0.501, p=9 \cdot 10^{-24}(68$ years $) ; \mu=0.17, \sigma=0.53, \mathrm{KS}$ statistic $=0.501$, $p=1 \cdot 10^{-23}(70$ years $) ; \mu=0.17, \sigma=0.53, \mathrm{KS}$ statistic $=0.501, p=1 \cdot 10^{-23}(71$ years $)$.

B) Fitting parameters for loglogistic: $a=1.20, b=2.86$, KS statistic $=0.838, p=5 \cdot 10^{-79}(5$ months); $a=1.23, b=3.13, \mathrm{KS}$ statistic $=0.850, p=1 \cdot 10^{-82}(2$ years $) ; a=1.26, b=3.33, \mathrm{KS}$ statistic $=0.862, p=3 \cdot 10^{-86}(23$ years $) ; a=1.26, b=3.23$, KS statistic $=0.867, p=7 \cdot 10^{-88}(27$ years); $a=1.26, b=3.33, \mathrm{KS}$ statistic $=0.863, p=2 \cdot 10^{-86}(38$ years $) ; a=1.23, b=3.23, \mathrm{KS}$ statistic $=0.846, p=3 \cdot 10^{-81}(45$ years $) ; a=1.22, b=3.33, \mathrm{KS}$ statistic $=0.837, p=8 \cdot 10^{-79}(57$ years); $a=1.21, b=3.23, \mathrm{KS}$ statistic $=0.828, p=3 \cdot 10^{-76}(58$ years $) ; a=1.21, b=3.23, \mathrm{KS}$ statistic $=0.835, p=3 \cdot 10^{-78}(68$ years $) ; a=1.22, b=3.23$, KS statistic $=0.839, p=2 \cdot 10^{-79}(70$ years); $a=1.21, b=3.23, \mathrm{KS}$ statistic $=0.834, p=4 \cdot 10^{-78}$ (71 years).

C) Fitting parameters for gamma: $\alpha=3.35, \beta=2.48$, KS statistic $=0.854, p=8 \cdot 10^{-84}(5$ months); $\alpha=3.84, \beta=2.83, \mathrm{KS}$ statistic $=0.865, p=2 \cdot 10^{-87}(2$ years $) ; \alpha=4.17, \beta=3.03, \mathrm{KS}$ statistic $=0.877, p=2 \cdot 10^{-91}(23$ years $) ; \alpha=4.07, \beta=2.93$, KS statistic $=0.883, p=1 \cdot 10^{-93}(27$ 
years); $\alpha=4.13, \beta=2.99, \mathrm{KS}$ statistic $=0.880, p=1 \cdot 10^{-93}(38$ years $) ; \alpha=4.06, \beta=2.99, \mathrm{KS}$ statistic $=0.868, p=4 \cdot 10^{-88}(45$ years $) ; \alpha=4.11, \beta=3.05$, KS statistic $=0.861, p=4 \cdot 10^{-86}(57$ years); $\alpha=4.03, \beta=3.03, \mathrm{KS}$ statistic $=0.853, p=2 \cdot 10^{-83}(58$ years $) ; \alpha=4.01, \beta=2.99, \mathrm{KS}$ statistic $=0.859, p=2 \cdot 10^{-85}(68$ years $) ; \alpha=4.07, \beta=3.02$, KS statistic $=0.863, p=2 \cdot 10^{-86}(70$ years); $\alpha=4.07, \beta=3.04, \mathrm{KS}$ statistic $=0.859, p=3 \cdot 10^{-85}$ (71 years).

Fig. 4

Similarity of spine head diameter distributions across human lifespan. Empirical data for human hippocampal spine head diameter (rectangles; taken from Das et al (2019)) ranging from infancy, through maturity, to senility look very similar. These data were fitted to three different distributions (solid lines). A) Fitting parameters for lognormal: $\mu=-1.08, \sigma=0.47$, KS statistic $=0.500, p=$ $1 \cdot 10^{-23}$ (5 months); $\mu=-1.07, \sigma=0.48, \mathrm{KS}$ statistic $=0.500, p=1 \cdot 10^{-23}(2$ years $) ; \mu=-1.05$ $\sigma=0.47, \mathrm{KS}$ statistic $=0.500, p=1 \cdot 10^{-23}(23$ years $) ; \mu=-1.05, \sigma=0.49, \mathrm{KS}$ statistic $=0.500$ $p=1 \cdot 10^{-23}(27$ years $) ; \mu=-1.06, \sigma=0.49, \mathrm{KS}$ statistic $=0.500, p=1 \cdot 10^{-23}(38$ years $) ; \mu=-1.05$ $\sigma=0.48, \mathrm{KS}$ statistic $=0.500, p=1 \cdot 10^{-23}(45$ years $) ; \mu=-1.05, \sigma=0.48, \mathrm{KS}$ statistic $=0.500$, $p=1 \cdot 10^{-23}(57$ years $) ; \mu=-1.06, \sigma=0.48, \mathrm{KS}$ statistic $=0.500, p=1 \cdot 10^{-23}(58$ years $) ; \mu=-1.06$, $\sigma=0.48, \mathrm{KS}$ statistic $=0.500, p=1 \cdot 10^{-23}(68$ years $) ; \mu=-1.06, \sigma=0.49, \mathrm{KS}$ statistic $=0.500$ $p=1 \cdot 10^{-23}(70$ years $) ; \mu=-1.06, \sigma=0.49, \mathrm{KS}$ statistic $=0.500, p=1 \cdot 10^{-23}(71$ years $)$.

B) Fitting parameters for loglogistic: $a=0.35, b=3.85$, KS statistic $=0.371, p=8 \cdot 10^{-13}(5$ months); $a=0.35, b=3.70, \mathrm{KS}$ statistic $=0.394, p=2 \cdot 10^{-14}(2$ years $) ; a=0.35, b=3.85, \mathrm{KS}$ statistic $=0.398, p=8 \cdot 10^{-15}(23$ years $) ; a=0.36, b=3.70, \mathrm{KS}$ statistic $=0.407, p=2 \cdot 10^{-15}(27$ years); $a=0.35, b=3.70, \mathrm{KS}$ statistic $=0.464, p=3 \cdot 10^{-20}(38$ years $) ; a=0.36, b=3.70, \mathrm{KS}$ statistic $=0.473, p=5 \cdot 10^{-21}(45$ years $) ; a=0.36, b=3.85$, KS statistic $=0.480, p=1 \cdot 10^{-21}(57$ years); $a=0.35, b=3.85, \mathrm{KS}$ statistic $=0.480, p=1 \cdot 10^{-21}(58$ years $) ; a=0.35, b=3.70, \mathrm{KS}$ 
statistic $=0.471, p=8 \cdot 10^{-21}(68$ years $) ; a=0.35, b=3.70, \mathrm{KS}$ statistic $=0.561, p=3 \cdot 10^{-30}(70$ years); $a=0.35, b=3.70, \mathrm{KS}$ statistic $=0.558, p=8 \cdot 10^{-30}$ (71 years).

C) Fitting parameters for gamma: $\alpha=5.03, \beta=13.44$, KS statistic $=0.372, p=6 \cdot 10^{-13}(5$ months); $\alpha=4.82, \beta=12.68, \mathrm{KS}$ statistic $=0.387, p=5 \cdot 10^{-14}$ (2 years); $\alpha=5.01, \beta=12.98, \mathrm{KS}$ statistic $=0.388, p=4 \cdot 10^{-14}(23$ years $) ; \alpha=4.77, \beta=12.19$, KS statistic $=0.397, p=9 \cdot 10^{-15}(27$ years $) ; \alpha=4.59, \beta=11.80, \mathrm{KS}$ statistic $=0.454, p=2 \cdot 10^{-19}(38$ years $) ; \alpha=4.80, \beta=12.27, \mathrm{KS}$ statistic $=0.469, p=1 \cdot 10^{-20}$ (45 years); $\alpha=4.90, \beta=12.55, \mathrm{KS}$ statistic $=0.475, p=3 \cdot 10^{-21}(57$ years); $\alpha=4.88, \beta=12.68, \mathrm{KS}$ statistic $=0.478, p=2 \cdot 10^{-21}(58$ years $) ; \alpha=4.73, \beta=12.21, \mathrm{KS}$ statistic $=0.466, p=2 \cdot 10^{-20}(68$ years $) ; \alpha=4.63, \beta=11.96, \mathrm{KS}$ statistic $=0.554, p=2 \cdot 10^{-29}(70$ years); $\alpha=4.59, \beta=11.79, \mathrm{KS}$ statistic $=0.551, p=5 \cdot 10^{-29}$ (71 years).

Fig. 5

Information encoded in spine volume is nearly optimal across brains with different sizes, regions, and physiological conditions. Note a nearly perfect alignment of the data points to the maximal entropy curve, especially for lognormal and loglogistic distributions. The gamma distribution has several outliers, which are rather mathematical artifacts. The visible outlier for all three distributions is the point corresponding to rat cerebellum. Legend for data points: diamonds for mouse, circles for rat, squares for macaque monkey, triangles for human.

Fig. 6

Information encoded in spine surface area is nearly optimal across mammalian brains with different regions and conditions. Similar as in Fig. 5, the nearly optimal alignment of data points to the maximal entropy curve, with a cerebellum as an outlier. Legend for data points: diamonds for mouse, circles for rat, pentagram for cat, squares for macaque monkey, triangles for human. 
Fig. 7

Information encoded in spine length is suboptimal. Note that the empirical entropies are generally suboptimal (especially for the gamma distribution), but nevertheless they are relatively close to the maximal entropy curve. Legend for data points: diamonds for mouse, circles for rat, pentagram for cat, squares for macaque monkey, triangles for human.

Fig. 8

Information encoded in spine head diameter is suboptimal. Note scattered data points, far away from the maximal entropy curve. Legend for data points: diamonds for mouse, circles for rat, stars for rabbit, plus for echidna, pentagram for cat, squares for macaque monkey, hexagon for dolphin, triangles for human.

Fig. 9

Density of information stored in dendritic spines is far from optimal. Density of entropy $F$ as a function of (A) spine volume, (B) spine surface area, (C) spine length, and (D) spine head diameter. Blue diamonds correspond to data points described by lognormal distribution, black squares correspond to loglogistic, and green triangles to gamma distribution. Note that all these data points are far below the theoretical upper bounds for the entropy density represented by three straight lines (blue solid line for lognormal, black dashed line for loglogistic, and green dotted line for gamma distribution). 
Table 1: Information contained in the distribution of spine volumes.

\begin{tabular}{|c|c|c|c|c|c|c|c|}
\hline $\begin{array}{l}\text { Species/ } \\
\text { region (cond.) }\end{array}$ & $\begin{array}{l}\text { Spine volume } \\
\text { mean } \pm \mathrm{SD} \\
\left(\mu \mathrm{m}^{3}\right)\end{array}$ & $\begin{array}{c}----- \\
H_{l n}\left(H_{l n, m}\right)\end{array}$ & $\begin{array}{c}\text { Entropy (bits) } \\
------- \\
H_{l l}\left(H_{l l, m}\right)\end{array}$ & $\begin{array}{r}----- \\
H_{g}\left(H_{g, m}\right) \\
\end{array}$ & $\begin{array}{r}\text { Effic } \\
---- \\
\eta_{\ln }\left(D_{\ln }\right)\end{array}$ & $\begin{array}{l}\text { ency } \% \text { (Devia } \\
------ \\
\eta_{l l}\left(D_{l l}\right)\end{array}$ & $\begin{array}{l}\text { ion } \%) \\
\eta_{g}\left(D_{g}\right)\end{array}$ \\
\hline \multicolumn{8}{|l|}{ Mouse } \\
\hline neoctx (wt) & $0.152 \pm 0.111^{a}$ & $8.37(8.57)$ & $8.22(8.48)$ & $8.55(8.69)$ & $99.1(25.9)$ & $96.9(47.8)$ & $98.4(87.5)$ \\
\hline neoctx (mut.) & $0.144 \pm 0.131^{a}$ & $8.42(8.50)$ & $8.25(8.40)$ & $8.60(8.61)$ & $99.1(16.8)$ & $98.1(34.5)$ & $99.8(20.8)$ \\
\hline neoctx & $0.081 \pm 0.087^{b}$ & $7.64(7.67)$ & $7.47(7.57)$ & $7.77(7.78)$ & $99.7(9.2)$ & $98.7(26.6)$ & $99.9(13.3)$ \\
\hline hippo. & $0.076 \pm 0.082^{c}$ & $7.55(7.57)$ & $7.38(7.48)$ & $7.68(7.69)$ & $99.7(8.9)$ & $98.7(26.4)$ & $99.9(14.1)$ \\
\hline hippo. & $0.038 \pm 0.036^{d}$ & $6.51(6.57)$ & $6.34(6.48)$ & $6.69(6.69)$ & $99.1(14.5)$ & $97.8(32.4)$ & $99.9(11.4)$ \\
\hline piriform 1a & $0.129 \pm 0.174^{d}$ & $8.34(8.34)$ & $8.19(8.25)$ & $8.23(8.45)$ & $99.9(1.4)$ & $99.4(18.3)$ & $97.4(45.0)$ \\
\hline piriform $1 b$ & $0.071 \pm 0.073^{d}$ & $7.44(7.48)$ & $7.27(7.38)$ & $7.59(7.59)$ & $99.5(11.1)$ & $98.5(28.5)$ & $99.9(5.4)$ \\
\hline somatosen. & $0.06 \pm 0.04^{e}$ & $6.97(7.23)$ & $6.82(7.14)$ & $7.13(7.35)$ & $96.3(28.6)$ & $95.6(54.5)$ & $97.0(125.0)$ \\
\hline visual & $0.09 \pm 0.07^{f}$ & $7.66(7.82)$ & $7.50(7.73)$ & $7.84(7.93)$ & $98.0(23.0)$ & $97.1(43.6)$ & $98.8(65.3)$ \\
\hline striat. (wt, 1m) & $0.050 \pm 0.075^{g}$ & $6.96(6.97)$ & $6.84(6.88)$ & $6.63(7.09)$ & $99.9(6.3)$ & $99.5(15.3)$ & $93.6(55.6)$ \\
\hline striat. (mut, 1m) & $0.077 \pm 0.100^{g}$ & $7.59(7.59)$ & $7.44(7.50)$ & $7.55(7.71)$ & $100.0(0.4)$ & $99.2(19.5)$ & $97.9(40.7)$ \\
\hline striat. (wt, 3m) & $0.074 \pm 0.094^{g}$ & $7.53(7.53)$ & $7.38(7.44)$ & $7.52(7.65)$ & $100.0(1.5)$ & $99.2(20.3)$ & $98.3(38.0)$ \\
\hline striat. (mut, $3 \mathrm{~m}$ ) & $0.089 \pm 0.162^{g}$ & $7.74(7.80)$ & $7.69(7.71)$ & $6.71(7.92)$ & $99.2(15.8)$ & $99.7(10.9)$ & $84.7(69.8)$ \\
\hline striat. (wt, 6m) & $0.084 \pm 0.115^{g}$ & $7.72(7.72)$ & $7.58(7.63)$ & $7.59(7.84)$ & $99.9(2.1)$ & $99.3(17.8)$ & $96.8(46.6)$ \\
\hline striat. (mut, $6 \mathrm{~m}$ ) & $0.088 \pm 0.143^{g}$ & $7.76(7.78)$ & $7.67(7.69)$ & $7.20(7.90)$ & $99.7(10.3)$ & $99.6(13.3)$ & $91.1(62.1)$ \\
\hline striat. (wt, 22m) & $0.143 \pm 0.211^{g}$ & $8.48(8.49)$ & $8.36(8.39)$ & $8.19(8.60)$ & $99.9(5.8)$ & $99.5(15.7)$ & $95.2(54.1)$ \\
\hline striat. (mut, 22m) & $0.089 \pm 0.146^{g}$ & $7.77(7.80)$ & $7.68(7.71)$ & $7.18(7.92)$ & $99.6(10.8)$ & $99.6(13.1)$ & $90.6(62.8)$ \\
\hline \multicolumn{8}{|l|}{ Rat } \\
\hline hippo. & $0.062 \pm 0.080^{h}$ & $7.28(7.28)$ & $7.13(7.19)$ & $7.24(7.40)$ & $100.0(0.7)$ & $99.2(19.7)$ & $97.9(39.9)$ \\
\hline hippo. (ctr) & $0.067 \pm 0.085^{i}$ & $7.39(7.39)$ & $7.24(7.30)$ & $7.38(7.51)$ & $99.9(1.5)$ & $99.1(20.3)$ & $98.2(37.9)$ \\
\hline hippo. (cLTP) & $0.080 \pm 0.101^{i}$ & $7.65(7.65)$ & $7.49(7.56)$ & $7.64(7.76)$ & $99.9(1.8)$ & $99.2(20.4)$ & $98.4(37.3)$ \\
\hline prefro. (ctr) & $0.09 \pm 0.18^{j}$ & $7.72(7.82)$ & $7.71(7.73)$ & $6.14(7.93)$ & $98.8(20.4)$ & $99.8(9.1)$ & $77.3(75.0)$ \\
\hline prefro. (stress) & $0.08 \pm 0.37^{j}$ & $6.94(7.65)$ & $7.55(7.56)$ & $-14.9(7.76)$ & $90.8(59.3)$ & $99.9(1.8)$ & $-(95.3)$ \\
\hline somatosen. (ctr) & $0.51 \pm 0.35^{k}$ & $10.08(10.32)$ & $9.93(10.23)$ & $10.24(10.44)$ & $97.6(32.6)$ & $97.1(52.3)$ & $98.2(112.3)$ \\
\hline somatosen. (LTP) & $0.79 \pm 0.49^{k}$ & $10.63(10.95)$ & $10.49(10.86)$ & $10.78(11.07)$ & $97.0(44.4)$ & $96.6(60.4)$ & $97.4(159.9)$ \\
\hline cerebel. & $0.12 \pm 0.02^{l}$ & $6.34(8.23)$ & $6.31(8.14)$ & $6.36(8.35)$ & $77.0(60.4)$ & $77.4(322.7)$ & $76.1(3500.0)$ \\
\hline \multicolumn{8}{|l|}{ Macaque } \\
\hline prefro. (young) & $0.106 \pm 0.660^{m}$ & $7.06(8.05)$ & $7.96(7.96)$ & $-37.4(8.17)$ & $87.6(73.6)$ & $99.9(0.9)$ & - $(97.4)$ \\
\hline prefro. (old) & $0.110 \pm 0.527^{m}$ & $7.37(8.11)$ & $8.02(8.02)$ & $-16.5(8.22)$ & $90.9(62.2)$ & $99.9(1.7)$ & $-(95.7)$ \\
\hline prefro. & $0.102 \pm 0.075^{n}$ & $7.80(8.00)$ & $7.65(7.91)$ & $7.98(8.12)$ & $97.6(25.2)$ & $96.7(47.4)$ & $98.3(85.0)$ \\
\hline visual (young) & $0.062 \pm 0.173^{m}$ & $6.99(7.28)$ & $7.18(7.18)$ & $1.70(7.40)$ & $96.1(35.9)$ & $99.9(4.9)$ & $23.0(87.2)$ \\
\hline visual (old) & $0.070 \pm 0.226^{m}$ & $7.06(7.45)$ & $7.36(7.36)$ & $-1.19(7.57)$ & $94.7(42.8)$ & $99.9(3.7)$ & $-(90.4)$ \\
\hline visual & $0.066 \pm 0.141^{n}$ & $7.24(7.37)$ & $7.27(7.28)$ & $5.17(7.49)$ & $98.3(23.3)$ & $99.8(8.1)$ & $69.0(78.1)$ \\
\hline cingul. & $0.07 \pm 0.15^{\circ}$ & $7.33(7.45)$ & $7.35(7.36)$ & $5.23(7.57)$ & $98.3(23.5)$ & $99.8(8.1)$ & $69.0(78.1)$ \\
\hline pariet. (young) & $0.196 \pm 0.353^{p}$ & $8.88(8.94)$ & $8.83(8.85)$ & $7.90(9.06)$ & $99.4(16.1)$ & $99.8(11.1)$ & $87.2(69.2)$ \\
\hline pariet. (old) & $0.255 \pm 1.528^{p}$ & $8.36(9.32)$ & $9.23(9.23)$ & $-32.8(9.44)$ & $89.7(80.5)$ & $99.9(0.9)$ & $-(97.2)$ \\
\hline \multicolumn{8}{|l|}{ Human } \\
\hline cingul. (40y api.) & $0.301 \pm 0.245^{r}$ & $9.43(9.56)$ & $9.26(9.47)$ & $9.61(9.68)$ & $98.6(22.7)$ & $97.8(40.8)$ & $99.3(50.9)$ \\
\hline cingul. (40y bas.) & $0.284 \pm 0.232^{r}$ & $9.34(9.48)$ & $9.18(9.38)$ & $9.53(9.59)$ & $98.6(22.4)$ & $97.8(40.6)$ & $99.3(49.9)$ \\
\hline cingul. (85y api.) & $0.310 \pm 0.244^{r}$ & $9.45(9.60)$ & 9. 里9 (9.51) & $9.63(9.72)$ & $98.4(24.2)$ & $97.7(42.9)$ & $99.1(61.4)$ \\
\hline cingul. ( $85 \mathrm{y}$ bas.) & $0.325 \pm 0.277^{r}$ & $9.56(9.67)$ & $9.40(9.58)$ & $9.75(9.79)$ & $98.9(20.9)$ & $98.1(38.1)$ & $99.6(37.7)$ \\
\hline
\end{tabular}

${ }^{a}$ Ishii et al (2018); ${ }^{b}$ Tamada et al $(2020) ;{ }^{c}$ Kashiwagi et al $(2019) ;{ }^{d}$ Schikorski and Stevens (1999); ${ }^{e}$ Rodriguez-Moreno et al (2018); ${ }^{f}$ Arellano et al (2007); ${ }^{g}$ Parajuli et al (2020); ${ }^{h}$ Harris and Stevens (1989); ${ }^{i}$ Borczyk (2019); ${ }^{j}$ Radley et al (2008); ${ }^{k}$ Cheetham et al (2014); ${ }^{l}$ Harris and Stevens (1988); ${ }^{m}$ Young et al (2014); ${ }^{n}$ Medalla and Luebke (2015); ${ }^{o}$ Medalla and Barbas (2009); ${ }^{p}$ Motley et al (2018) (and J.H. Morrison, private comm.); ${ }^{r}$ Benavides-Piccione (2013; and private comm.). 
Table 2: Information contained in the distribution of spine/PSD areas.

\begin{tabular}{|c|c|c|c|c|c|c|c|}
\hline $\begin{array}{l}\text { Species/ } \\
\text { region (cond.) }\end{array}$ & $\begin{array}{l}\text { Spine area } \\
\text { mean } \pm S D \\
\left(\mu \mathrm{m}^{2}\right)\end{array}$ & $\begin{array}{l}----- \\
H_{l n}\left(H_{l n, m}\right)\end{array}$ & $\begin{array}{l}\text { ntropy (bits) } \\
----- \\
H_{l l}\left(H_{l l, m}\right)\end{array}$ & $\begin{array}{l}----- \\
H_{g}\left(H_{g, m}\right)\end{array}$ & $\begin{array}{l}\text { Effici } \\
----- \\
\eta_{l n}\left(D_{l n}\right)\end{array}$ & $\begin{array}{l}\text { ncy \% (Devia } \\
------ \\
\eta_{l l}\left(D_{l l}\right)\end{array}$ & $\begin{array}{l}\text { on } \%) \\
----- \\
\eta_{g}\left(D_{g}\right)\end{array}$ \\
\hline \multicolumn{8}{|l|}{ Mouse } \\
\hline hippo. & $0.047 \pm 0.038^{a}$ & $3.42(3.56)$ & $3.26(3.47)$ & $3.61(3.68)$ & $96.1(21.1)$ & $94.0(41.2)$ & $98.1(53.0)$ \\
\hline hippo. (PSD) & $0.043 \pm 0.031^{b}$ & $3.22(3.43)$ & $3.07(3.34)$ & $3.40(3.55)$ & $93.9(25.6)$ & $91.9(48.7)$ & $95.7(92.4)$ \\
\hline piriform 1a (PSD) & $0.096 \pm 0.105^{b}$ & $4.57(4.59)$ & $4.40(4.50)$ & $4.69(4.71)$ & $99.6(8.4)$ & $97.9(25.8)$ & $99.7(16.4)$ \\
\hline piriform 1b (PSD) & $0.100 \pm 0.087^{b}$ & $4.55(4.65)$ & $4.38(4.56)$ & $4.73(4.76)$ & $97.9(18.5)$ & $96.2(36.9)$ & $99.3(32.1)$ \\
\hline visual (PSD) & $0.08 \pm 0.06^{c}$ & $4.14(4.33)$ & $3.99(4.23)$ & $4.32(4.44)$ & $95.8(24.3)$ & $94.2(46.0)$ & $97.3(77.8)$ \\
\hline visual & $0.31 \pm 0.35^{d}$ & $6.27(6.28)$ & $6.10(6.19)$ & $6.37(6.40)$ & $99.8(7.6)$ & $98.6(24.5)$ & $99.5(21.6)$ \\
\hline temporal & $0.37 \pm 0.36^{d}$ & $6.48(6.53)$ & $6.31(6.44)$ & $6.65(6.65)$ & $99.2(15.2)$ & $98.0(31.1)$ & $99.9(5.7)$ \\
\hline somatosen. & $0.87 \pm 0.47^{e}$ & $7.32(7.77)$ & $7.20(7.68)$ & $7.45(7.89)$ & $94.3(53.9)$ & $93.8(73.1)$ & $94.5(242.7)$ \\
\hline \multicolumn{8}{|l|}{ Rat } \\
\hline hippo. & $0.83 \pm 0.63^{f}$ & $7.53(7.70)$ & $7.37(7.61)$ & $7.71(7.82)$ & $97.7(36.3)$ & $96.8(45.2)$ & $98.6(73.6)$ \\
\hline hippo. (PSD) & $0.069 \pm 0.080^{f}$ & $4.10(4.11)$ & $3.94(4.02)$ & $4.18(4.23)$ & $99.8(5.7)$ & $98.0(23.5)$ & $98.9(25.6)$ \\
\hline hippo. (PSD; ctr) & $0.068 \pm 0.073^{g}$ & $4.07(4.09)$ & $3.90(4.00)$ & $4.20(4.21)$ & $99.4(9.2)$ & $97.5(26.7)$ & $99.8(13.2)$ \\
\hline hippo. (PSD; LTP) & $0.109 \pm 0.179^{g}$ & $4.74(4.77)$ & $4.65(4.68)$ & $4.14(4.89)$ & $99.4(10.9)$ & $99.4(13.0)$ & $84.7(62.9)$ \\
\hline prefro. (ctr) & $1.1 \pm 1.8^{h}$ & $8.08(8.11)$ & $7.99(8.02)$ & $7.49(8.22)$ & $99.7(28.2)$ & $99.6(13.1)$ & $91.1(62.7)$ \\
\hline prefro. (stress) & $1.0 \pm 1.9^{h}$ & $7.89(7.97)$ & $7.86(7.88)$ & $6.63(8.09)$ & $99.1(40.9)$ & $99.8(10.0)$ & $82.0(72.3)$ \\
\hline forebr. (PSD; E19d) & $0.195 \pm 0.118^{i}$ & $5.27(5.61)$ & $5.14(5.52)$ & $5.42(5.73)$ & $93.9(33.2)$ & $93.0(62.5)$ & $94.5(173.1)$ \\
\hline forebr. (PSD; P2d) & $0.313 \pm 0.216^{i}$ & $6.05(6.29)$ & $5.91(6.20)$ & $6.22(6.41)$ & $96.2(29.6)$ & $95.2(51.9)$ & $97.1(110.0)$ \\
\hline forebr. (PSD; P21d) & $0.259 \pm 0.214^{i}$ & $5.90(6.02)$ & $5.73(5.93)$ & $6.08(6.14)$ & $97.9(21.7)$ & $96.7(39.9)$ & $99.1(46.5)$ \\
\hline forebr. (PSD; P60d) & $0.251 \pm 0.153^{i}$ & $5.64(5.97)$ & $5.50(5.88)$ & $5.79(6.09)$ & $94.3(33.5)$ & $93.6(61.9)$ & $95.0(169.1)$ \\
\hline cerebel. & $1.12 \pm 0.18^{j}$ & $6.19(8.13)$ & $6.16(8.04)$ & $6.21(8.25)$ & $76.1(107.1)$ & $76.6(336.7)$ & $75.2(3771.6)$ \\
\hline cerebel. (PSD) & $0.15 \pm 0.08^{j}$ & $4.77(5.23)$ & $4.66(5.14)$ & $4.90(5.35)$ & $91.2(37.0)$ & $90.6(74.4)$ & $91.6(251.6)$ \\
\hline \multicolumn{8}{|l|}{ Cat } \\
\hline visual & $0.099 \pm 0.046^{k}$ & $4.04(4.63)$ & $3.94(4.54)$ & $4.14(4.75)$ & $87.1(40.7)$ & $86.6(89.7)$ & $87.1(363.2)$ \\
\hline \multicolumn{8}{|l|}{ Macaque } \\
\hline prefro. (PSD) & $0.12 \pm 0.13^{l}$ & $4.89(4.91)$ & $4.72(4.82)$ & $5.01(5.03)$ & $99.6(8.9)$ & $98.0(26.2)$ & $99.7(14.8)$ \\
\hline visual (PSD) & $0.08 \pm 0.18^{l}$ & $4.17(4.33)$ & $4.22(4.23)$ & $1.64(4.44)$ & $96.4(26.0)$ & $99.8(7.4)$ & $37.0(80.3)$ \\
\hline cingul. (PSD) & $0.13 \pm 0.31^{m}$ & $4.84(5.03)$ & $4.93(4.94)$ & $1.72(5.14)$ & $96.3(29.6)$ & $99.8(6.6)$ & $33.3(82.4)$ \\
\hline \multicolumn{8}{|l|}{ Human } \\
\hline neoctx (PSD) & $0.205 \pm 0.121^{n}$ & $5.32(5.68)$ & $5.19(5.59)$ & $5.46(5.80)$ & $93.6(34.2)$ & $92.8(64.7)$ & $94.2(187.0)$ \\
\hline temporal & $0.59 \pm 0.53^{d}$ & $7.12(7.21)$ & $6.96(7.12)$ & $7.31(7.33)$ & $98.8(21.6)$ & $97.7(35.1)$ & $99.7(23.9)$ \\
\hline
\end{tabular}

${ }^{a}$ Santuy et al $(2020) ;{ }^{b}$ Schikorski and Stevens $(1999) ;{ }^{c}$ Arellano et al (2007); ${ }^{d}$

Benavides-Piccione et al (2002); ${ }^{e}$ Rodriguez-Moreno et al (2018); ${ }^{f}$ Harris and

Stevens (1989); ${ }^{g}$ Borczyk et al (2019); ${ }^{h}$ Radley et al (2008); ${ }^{i}$ Swulius et al (2010); ${ }^{j}$ Harris and Stevens (1988); ${ }^{k}$ da Costa (2013); ${ }^{l}$ Medalla and Luebke (2015); ${ }^{m}$ Medalla and Barbas (2009); ${ }^{n}$ Tang et al (2001). 
Table 3: Information contained in the distribution of spine length.

\begin{tabular}{|c|c|c|c|c|c|c|c|}
\hline $\begin{array}{l}\text { Species/ } \\
\text { region (cond.) }\end{array}$ & $\begin{array}{l}\text { Spine length } \\
\text { mean } \pm \mathrm{SD} \\
(\mu \mathrm{m})\end{array}$ & $\begin{array}{l}----- \\
H_{l n}\left(H_{l n, m}\right)\end{array}$ & $\begin{array}{l}\text { Intropy (bits } \\
----- \\
H_{l l}\left(H_{l l, m}\right)\end{array}$ & $\begin{array}{l}H_{g}\left(H_{g, m}\right) \\
-\end{array}$ & $\begin{array}{l}\text { Effici } \\
----- \\
\eta_{l n}\left(D_{l n}\right)\end{array}$ & $\begin{array}{l}\text { ncy \% (Devia } \\
------ \\
\eta_{l l}\left(D_{l l}\right)\end{array}$ & $\begin{array}{l}\text { ion } \%) \\
\eta_{g}\left(D_{g}\right)\end{array}$ \\
\hline \multicolumn{8}{|l|}{ Mouse } \\
\hline neoctx & $0.836 \pm 0.569^{a}$ & $4.14(4.39)$ & $3.99(4.30)$ & $4.31(4.51)$ & $94.3(42.1)$ & $92.9(52.9)$ & $95.6(115.9)$ \\
\hline hippo. & $0.95 \pm 0.42^{b}$ & $3.92(4.57)$ & $3.83(4.48)$ & $4.02(4.69)$ & $85.8(66.7)$ & $85.4(95.9)$ & $85.7(411.6)$ \\
\hline hippo. & $0.83 \pm 0.27^{c}$ & $3.37(4.38)$ & $3.30(4.29)$ & $3.43(4.50)$ & $77.0(66.9)$ & $77.1(143.0)$ & $76.2(845.0)$ \\
\hline hippo. (neck) & $0.42 \pm 0.24^{c}$ & $3.00(3.40)$ & $2.88(3.31)$ & $3.14(3.51)$ & $88.4(38.0)$ & $87.1(67.7)$ & $89.4(206.2)$ \\
\hline visual (neck) & $0.66 \pm 0.37^{d}$ & $3.64(4.05)$ & $3.51(3.96)$ & $3.77(4.17)$ & $89.8(43.9)$ & $88.8(69.5)$ & $90.5(218.2)$ \\
\hline striat. (wt, 1m) & $1.00 \pm 0.52^{e}$ & $4.16(4.65)$ & $4.05(4.56)$ & $4.29(4.76)$ & $89.6(64.8)$ & $88.9(77.1)$ & $90.0(269.8)$ \\
\hline striat. (mut, $1 \mathrm{~m}$ ) & $1.14 \pm 0.57^{e}$ & $4.31(4.84)$ & $4.21(4.75)$ & $4.43(4.95)$ & $89.2(83.3)$ & $88.6(81.3)$ & $89.4(299.9)$ \\
\hline striat. (wt, 3m) & $1.13 \pm 0.57^{e}$ & $4.31(4.82)$ & $4.20(4.73)$ & $4.43(4.94)$ & $89.4(81.3)$ & $88.8(80.3)$ & $89.6(293.0)$ \\
\hline striat. (mut, $3 \mathrm{~m}$ ) & $1.11 \pm 0.57^{e}$ & $4.30(4.80)$ & $4.19(4.71)$ & $4.42(4.92)$ & $89.7(77.6)$ & $89.0(78.4)$ & $90.0(279.2)$ \\
\hline striat. (wt, 6m) & $0.98 \pm 0.56^{e}$ & $4.22(4.62)$ & $4.10(4.53)$ & $4.36(4.74)$ & $91.5(58.9)$ & $90.5(67.7)$ & $92.1(206.3)$ \\
\hline striat. (mut, $6 \mathrm{~m}$ ) & $1.21 \pm 0.63^{e}$ & $4.44(4.92)$ & $4.33(4.83)$ & $4.56(5.04)$ & $90.2(94.1)$ & $89.6(76.9)$ & $90.5(268.9)$ \\
\hline striat. (wt, 22m) & $1.03 \pm 0.54^{e}$ & $4.22(4.69)$ & $4.10(4.60)$ & $4.34(4.81)$ & $89.9(67.3)$ & $89.2(76.2)$ & $90.2(263.8)$ \\
\hline striat. (mut, 22m) & $1.11 \pm 0.57^{e}$ & $4.30(4.80)$ & $4.19(4.71)$ & $4.42(4.92)$ & $89.7(77.6)$ & $89.0(78.4)$ & $90.0(279.2)$ \\
\hline \multicolumn{8}{|l|}{ Rat } \\
\hline hippo. & $0.95 \pm 0.42^{f}$ & $3.92(4.57)$ & $3.83(4.48)$ & $4.02(4.69)$ & $85.8(66.7)$ & $85.4(95.9)$ & $85.7(411.6)$ \\
\hline prefro. (ctr) & $0.55 \pm 1.80^{g}$ & $3.38(3.78)$ & $3.69(3.69)$ & $-5.21(3.90)$ & $89.3(61.9)$ & $99.9(3.6)$ & $-(90.7)$ \\
\hline prefro. (stress) & $0.55 \pm 0.95^{g}$ & $3.74(3.78)$ & $3.67(3.69)$ & $2.95(3.90)$ & $98.9(17.5)$ & $99.3(11.9)$ & $75.5(66.5)$ \\
\hline cerebel. & $1.22 \pm 0.30^{h}$ & $3.57(4.93)$ & $3.52(4.84)$ & $3.60(5.05)$ & $72.3(122.8)$ & $72.7(202.8)$ & $71.3(1553.8)$ \\
\hline \multicolumn{8}{|l|}{ Cat } \\
\hline visual & $1.03 \pm 0.74^{i}$ & $4.48(4.69)$ & $4.33(4.60)$ & $4.65(4.81)$ & $95.5(50.6)$ & $94.1(49.0)$ & $96.8(93.8)$ \\
\hline \multicolumn{8}{|l|}{ Macaque } \\
\hline prefro. (young) & $1.481 \pm 2.968^{j}$ & $5.12(5.21)$ & $5.11(5.12)$ & $3.52(5.33)$ & $98.1(202.8)$ & $99.7(9.1)$ & $66.0(75.1)$ \\
\hline prefro. (old) & $1.336 \pm 3.516^{j}$ & $4.82(5.07)$ & $4.97(4.97)$ & $0.44(5.18)$ & $95.1(182.6)$ & $99.9(5.5)$ & $8.5(85.6)$ \\
\hline visual (young) & $1.388 \pm 3.179^{j}$ & $4.96(5.12)$ & $5.02(5.03)$ & $2.26(5.24)$ & $96.8(172.7)$ & $99.8(7.1)$ & $43.1(81.0)$ \\
\hline visual (old) & $1.462 \pm 2.883^{j}$ & $5.10(5.20)$ & $5.09(5.10)$ & $3.61(5.31)$ & $98.3(173.6)$ & $99.7(9.4)$ & $68.0(74.3)$ \\
\hline visual (young) & $1.10 \pm 1.32^{k}$ & $4.78(4.78)$ & $4.62(4.69)$ & $4.83(4.90)$ & $99.9(10.3)$ & $98.4(22.3)$ & $98.5(30.5)$ \\
\hline visual (old) & $1.20 \pm 2.43^{k}$ & $4.81(4.91)$ & $4.81(4.82)$ & $3.14(5.03)$ & $97.9(72.7)$ & $99.7(9.0)$ & $62.4(75.6)$ \\
\hline \multicolumn{8}{|l|}{ Human } \\
\hline cingul. (40y api.) & $1.780 \pm 0.740^{l}$ & $4.76(5.48)$ & $4.67(5.39)$ & $4.85(5.60)$ & $86.9(390.2)$ & $86.7(104.0)$ & $86.6(478.6)$ \\
\hline cingul. (40y bas.) & $1.417 \pm 0.710^{l}$ & $4.63(5.15)$ & $4.52(5.06)$ & $4.75(5.27)$ & $89.9(184.9)$ & $89.4(81.0)$ & $90.1(298.3)$ \\
\hline cingul. (85y api.) & $1.709 \pm 0.769^{l}$ & $4.79(5.42)$ & $4.69(5.33)$ & $4.89(5.54)$ & $88.5(804.1)$ & $88.0(93.7)$ & $88.3(393.9)$ \\
\hline cingul. ( $85 \mathrm{y}$ bas.) & $1.483 \pm 0.715^{l}$ & $4.66(5.22)$ & $4.55(5.13)$ & $4.77(5.33)$ & $89.3(266.8)$ & $88.8(85.4)$ & $89.4(330.2)$ \\
\hline prefro. (non-alzh.) & $1.219 \pm 2.335^{m}$ & $4.85(4.93)$ & $4.82(4.84)$ & $3.54(5.05)$ & $98.4(65.6)$ & $99.6(9.9)$ & $70.1(72.7)$ \\
\hline prefro. (alzh.) & $1.360 \pm 2.980^{m}$ & $4.95(5.09)$ & $4.99(5.00)$ & $2.66(5.21)$ & $97.3(141.1)$ & $99.8(7.7)$ & $51.2(79.2)$ \\
\hline hippo. (5m) & $1.347 \pm 0.697^{n}$ & $4.59(5.08)$ & $4.48(4.99)$ & $4.71(5.19)$ & $90.4(138.3)$ & $89.8(77.6)$ & $90.7(273.5)$ \\
\hline hippo. (2y) & $1.359 \pm 0.662^{n}$ & $4.54(5.09)$ & $4.44(5.00)$ & $4.65(5.21)$ & $89.2(148.9)$ & $88.7(84.2)$ & $89.4(321.4)$ \\
\hline hippo. (23y) & $1.376 \pm 0.650^{n}$ & $4.53(5.11)$ & $4.43(5.02)$ & $4.63(5.23)$ & $88.6(160.9)$ & $88.2(87.8)$ & $88.7(348.1)$ \\
\hline hippo. (27y) & $1.390 \pm 0.669^{n}$ & $4.56(5.12)$ & $4.46(5.03)$ & $4.67(5.24)$ & $89.1(168.4)$ & $88.6(85.6)$ & $89.1(331.7)$ \\
\hline hippo. (38y) & $1.383 \pm 0.663^{n}$ & $4.55(5.12)$ & $4.453(5.02)$ & $4.66(5.23)$ & $89.0(164.1)$ & $88.5(86.0)$ & $89.0(335.1)$ \\
\hline hippo. (45y) & $1.358 \pm 0.659^{n}$ & $4.54(5.09)$ & $4.43(5.00)$ & $4.65(5.21)$ & $89.2(148.7)$ & $88.7(84.6)$ & $89.3(324.6)$ \\
\hline hippo. (57y) & $1.346 \pm 0.651^{n}$ & $4.52(5.08)$ & $4.42(4.99)$ & $4.63(5.19)$ & $89.1(142.9)$ & $88.6(85.0)$ & $89.2(327.5)$ \\
\hline hippo. (58y) & $1.331 \pm 0.649^{n}$ & $4.51(5.06)$ & $4.41(4.97)$ & $4.62(5.18)$ & $89.2(135.4)$ & $88.7(84.1)$ & $89.3(320.6)$ \\
\hline hippo. (68y) & $1.342 \pm 0.655^{n}$ & $4.53(5.07)$ & $4.42(4.98)$ & $4.64(5.19)$ & $89.2(140.3)$ & $88.7(84.0)$ & $89.4(319.8)$ \\
\hline hippo. (70y) & $1.348 \pm 0.654^{n}$ & $4.53(5.08)$ & $4.42(4.99)$ & $4.64(5.20)$ & $89.1(143.6)$ & $88.6(84.7)$ & $89.2(324.8)$ \\
\hline hippo. (71y) & $1.341 \pm 0.649^{n}$ & $4.52(5.07)$ & $4.41(4.98)$ & $4.63(5.19)$ & $89.1(140.4)$ & $88.6(84.9)$ & $89.2(326.9)$ \\
\hline
\end{tabular}

${ }^{a}$ Tamada et al (2020); ${ }^{b}$ Kashiwagi et al (2019); ${ }^{c}$ Scheuss and Bonhoeffer (2014); ${ }^{d}$ Arellano et al (2007); ${ }^{e}$ Parajuli et al $(2020){ }^{f}$ Harris and Stevens (1989); ${ }^{g}$ Radley et al (2008); ${ }^{h}$ Harris and Stevens (1988); ${ }^{i}$ da Costa (2013); $j$ Young et al (2014); ${ }^{k}$ Luebke et al (2015); ${ }^{l}$ Benavides-Piccione (2013; and private comm.); ${ }^{m}$ Boros et al (2019; and J.H. Herskowitz, private comm.) ; ${ }^{n}$ Das et al (2019; and S.C. Das, private comm.). 
Table 4: Information contained in the distribution of spine head/PSD diameter.

\begin{tabular}{|c|c|c|c|c|c|c|c|}
\hline $\begin{array}{l}\text { Species/ } \\
\text { region (cond.) }\end{array}$ & $\begin{array}{l}\text { Spine diameter } \\
\text { mean } \pm \mathrm{SD} \\
(\mu \mathrm{m})\end{array}$ & $\begin{array}{l}------ \\
H_{l n}\left(H_{l n, m}\right)\end{array}$ & $\begin{array}{l}\text { ntropy (bits } \\
----- \\
H_{l l}\left(H_{l l, m}\right)\end{array}$ & $\begin{array}{l}----- \\
H_{g}\left(H_{g, m}\right)\end{array}$ & $\begin{array}{r}\text { Effi } \\
---- \\
\eta_{\ln }\left(D_{\ln }\right)\end{array}$ & $\begin{array}{l}\text { ncy \% (Devia } \\
------ \\
\eta_{l l}\left(D_{l l}\right)\end{array}$ & $\begin{array}{l}\text { ion \%) } \\
------ \\
\eta_{g}\left(D_{g}\right)\end{array}$ \\
\hline \multicolumn{8}{|l|}{ Mouse } \\
\hline hippo. & $0.66 \pm 0.11^{a}$ & $2.15(4.05)$ & $2.12(3.96)$ & $2.17(4.17)$ & $53.2(69.9)$ & $53.6(322.7)$ & $52.2(3500.0)$ \\
\hline visual & $0.42 \pm 0.13^{b}$ & $2.33(3.40)$ & $2.26(3.31)$ & $2.38(3.51)$ & $68.5(54.6)$ & $68.5(152.3)$ & $67.7(943.8)$ \\
\hline
\end{tabular}

Rat

hippo.

cerebel.

$0.53 \pm 0.28^{c}$

$0.57 \pm 0.05^{d}$

$3.26(3.73)$

$3.15(3.64) \quad 3.39(3.85)$

$1.04(3.84)$

$1.07(3.75) \quad 1.06(3.95)$

$87.5(42.7)$

$27.1(875.5)$

$86.5(75.4)$

$28.5(637.6)$

$88.0(258.3)$

Rabbit

hippo. (E21d)

hippo. (E24d)

hippo. (E28d)

hippo. (P2d)

hippo. (P8d)

hippo. (P14d)

hippo. (adult)

$0.33 \pm 0.09^{e}$
$0.49 \pm 0.13^{e}$
$0.55 \pm 0.15^{e}$
$0.48 \pm 0.11^{e}$
$0.43 \pm 0.10^{e}$
$0.42 \pm 0.11^{e}$
$0.41 \pm 0.10^{e}$

$0.323 \pm 0.120^{f}$

$2.17(3.02)$

$2.09(2.93)$

$2.24(3.13)$

$71.9(49.1)$

$71.5(120.5)$

$71.5(624.5)$

\section{Cat}

auditory (hear)

auditory (deaf)

$0.672 \pm 0.213^{g}$

$0.707 \pm 0.233^{g}$

$3.03(4.07)$

2.97 (3.98)

3.16 (4.15)

3.09 (4.06)

3.09 (4.19)

3.21 (4.26)

$74.5(60.4)$

74.6 (147.8)

76.1 (140.6)

73.7 (895.4)

$75.4(820.7)$

\section{Macaque}

prefro. (young)

prefro. (old)

visual (young)

visual (old)

visual (young)

visual (old)

$0.403 \pm 0.660^{h}$
$0.420 \pm 0.440^{h}$
$0.376 \pm 0.289^{h}$
$0.383 \pm 0.226^{h}$
$0.43 \pm 0.53^{i}$
$0.46 \pm 0.30^{i}$

$3.31(3.34)$

$3.37(3.40)$

$3.07(3.24)$

$2.90(3.26)$

$3.43(3.43)$

$3.24(3.53)$

$3.22(3.25)$

$3.20(3.31)$

$2.72(3.45)$

$2.91(3.15) \quad 3.25(3.35)$

$2.77(3.17) \quad 3.04(3.38)$

$3.27(3.34) \quad 3.45(3.55)$

$3.10(3.44) \quad 3.40(3.64)$

$99.2(12.6)$

99.1 (11.9)

$94.8(25.9)$

$88.8(36.3)$

$99.9(3.4)$

$92.0(33.7)$

$2.37(3.14)$

$2.29(3.05)$

$2.45(3.26)$

$75.5(47.8)$

\section{Human}

neoctx

temporal

prefro. (non-alzh.)

prefro. (alzh.)

hippo. (5m)

hippo. $(2 \mathrm{y})$

hippo. (23y)

hippo. (27y)

hippo. (38y)

hippo. (45y)

hippo. (57y)

hippo. (58y)

hippo. (68y)

hippo. (70y)

hippo. (71y)
$0.466 \pm 0.121^{k}$

$0.30 \pm 0.09^{l}$

$0.573 \pm 0.803^{m}$

$0.562 \pm 1.141^{m}$

$0.375 \pm 0.166^{n}$

$0.380 \pm 0.175^{n}$

$0.386 \pm 0.175^{n}$

$0.391 \pm 0.181^{n}$

$0.389 \pm 0.184^{n}$

$0.391 \pm 0.181^{n}$

$0.391 \pm 0.179^{n}$

$0.387 \pm 0.181^{n}$

$0.387 \pm 0.183^{n}$

$0.390 \pm 0.185^{n}$
$0.385 \pm 0.177^{n}$
$2.25(3.55)$

$1.80(2.91)$

$3.84(3.84)$

$3.71(3.82)$

$2.58(3.23)$

$2.64(3.25)$

$2.65(3.27)$

$2.69(3.29)$

$2.71(3.29)$

$2.69(3.29)$

$2.68(3.29)$

$2.66(3.27)$

$2.69(3.28)$

$2.70(3.28)$

$2.71(3.29)$
$2.20(3.45) \quad 2.29(3.66)$

$1.74(2.82) \quad 1.85(3.03)$

$3.71(3.75) \quad 3.67(3.96)$

$3.71(3.73) \quad 2.03(3.93)$

$2.49(3.14) \quad 2.68(3.35)$

$2.55(3.16) \quad 2.75(3.37)$

$2.55(3.18) \quad 2.75(3.39)$

$2.99(3.20) \quad 2.79(3.41)$

$2.60(3.19) \quad 2.81(3.40)$

$2.59(3.20) \quad 2.79(3.41)$

$2.58(3.20) \quad 2.78(3.41)$

$2.56(3.18) \quad 2.76(3.39)$

$2.59(3.19) \quad 2.79(3.40)$

$2.60(3.19) \quad 2.81(3.40)$

$2.61(3.20) \quad 2.82(3.41)$
$63.5(58.8)$

$61.9(53.4)$

$99.9(4.2)$

$97.3(28.6)$

$79.9(45.3)$

$81.3(44.2)$

$81.0(44.8)$

$81.7(44.2)$

$82.4(43.6)$

$81.7(44.2)$

$81.4(44.6)$

$81.4(44.3)$

$82.0(43.9)$

$82.3(43.5)$

$82.5(43.5)$
$75.0(110.3)$

$75.3(532.2)$

$99.1(13.1)$ $96.7(27.7)$

$92.6(44.4)$

$87.3(64.7)$

$98.0(21.3)$

$90.3(56.2)$

$78.7(62.7)$

$99.9(8.9)$

$96.9(69.3)$

$90.0(187.2)$

97.2 (34.2)

93.4 (135.1)

$63.7(189.7)$

61.8 (158.4)

$98.8(17.2)$

$99.6(8.9)$

$79.2(95.7)$

$80.5(90.8)$

$80.2(92.7)$

$81.0(90.2)$

$81.5(87.6)$

$81.0(90.2)$

$80.6(91.5)$

$80.6(91.0)$

$81.2(88.9)$

$81.5(87.6)$

$81.6(87.3)$
$62.5(1383.2)$ 61.1 (1011.1) 92.6 (49.1)

$51.5(75.7)$ $80.0(410.3)$ $81.5(371.5)$ 81.1 (386.5) $82.0(366.7)$ $82.7(346.9)$ $82.0(366.7)$ 81.6 (377.1) 81.6 (373.1) $82.2(357.2)$ $82.6(347.2)$ $82.8(344.4)$

${ }^{a}$ Scheuss and Bonhoeffer $(2014) ;{ }^{b}$ Konur et al (2003); ${ }^{c}$ Harris and Stevens (1989); ${ }^{d}$ Harris and Stevens (1988); ${ }^{e}$ Schwartzkroin (1982); ${ }^{f}$ Hassiotis et al (2003); ${ }^{g}$ Clemo et al (2016); ${ }^{h}$ Young et al $(2014) ;{ }^{i}$ Luebke et al $(2015) ;{ }^{j}$ Glezer and Morgane (1990); ${ }^{k}$ Tang et al (2001); ${ }^{l}$ Alonso-Nanclares et al (2008); ${ }^{m}$ Boros et al (2019; and private comm.) ${ }^{n}$ Das et al (2019; and private comm.). 

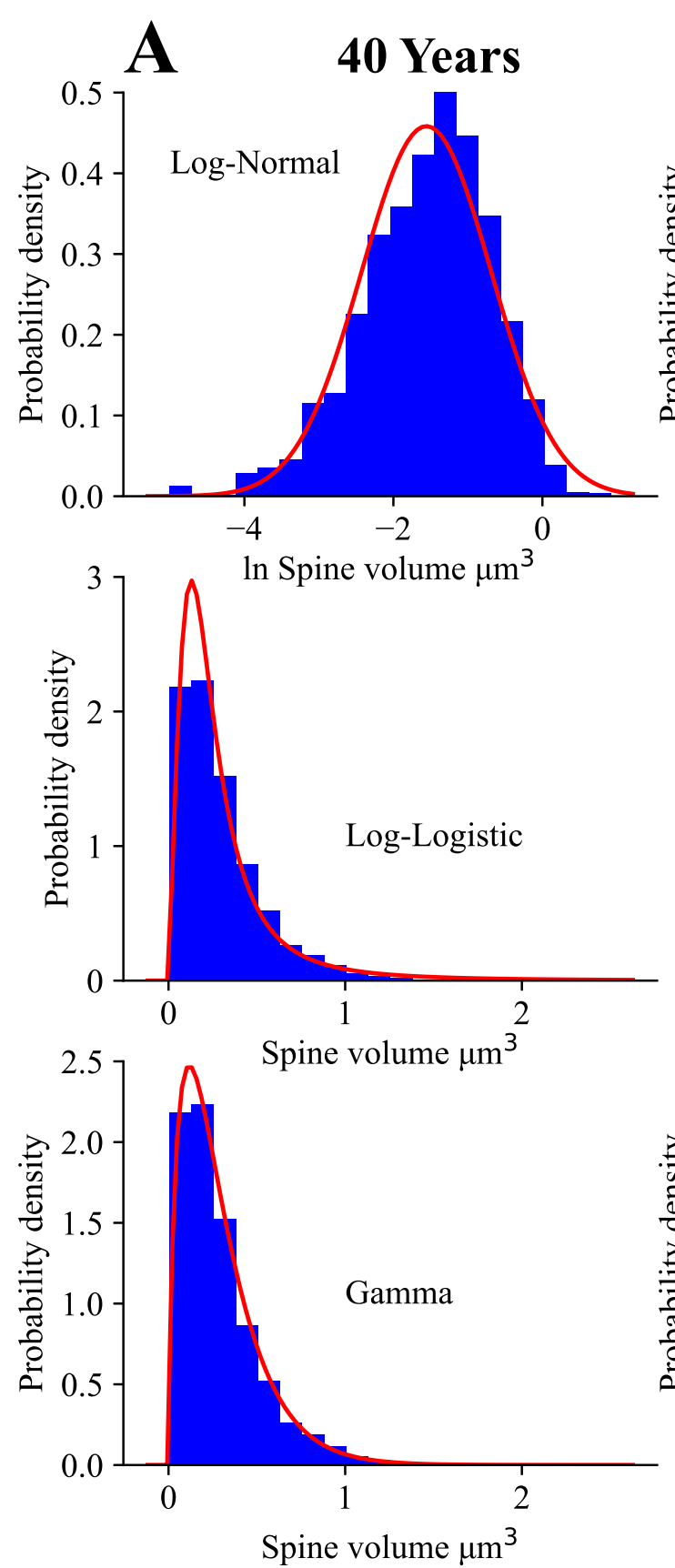
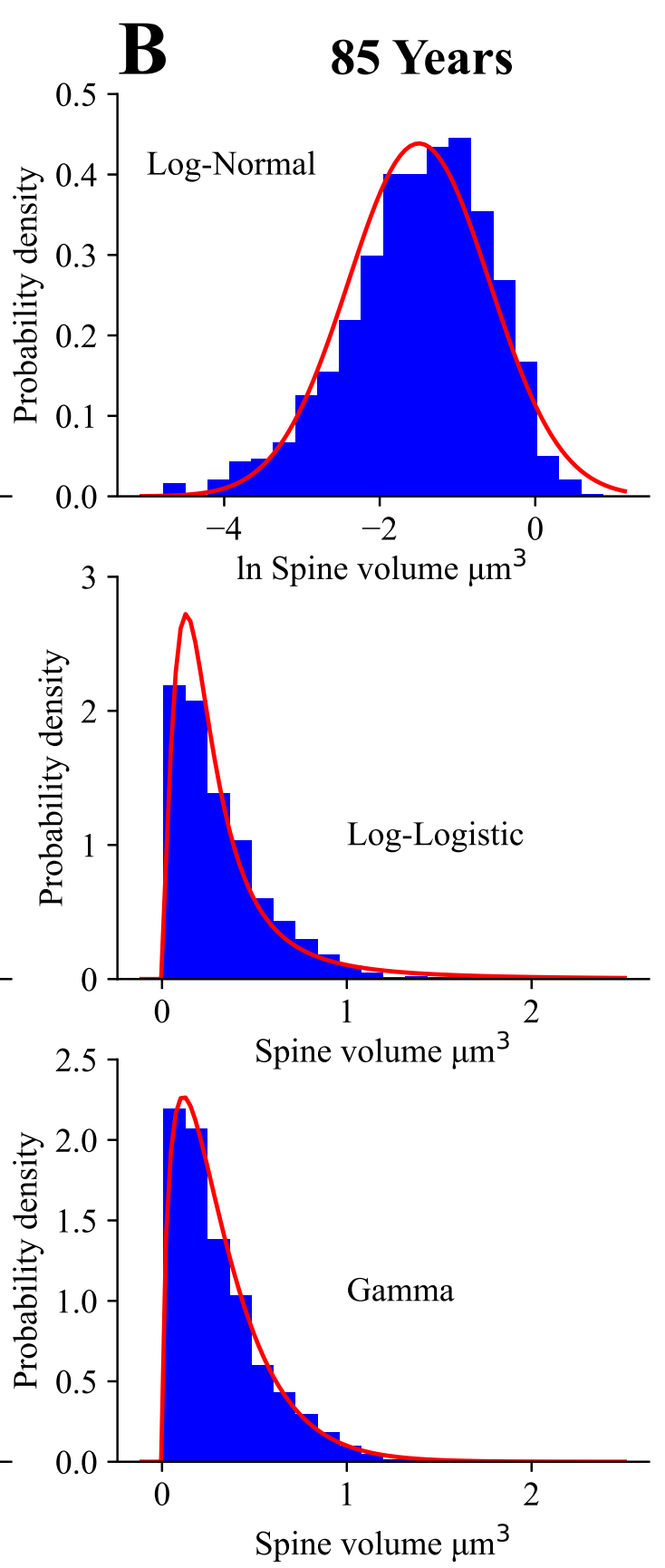

Figure 1 

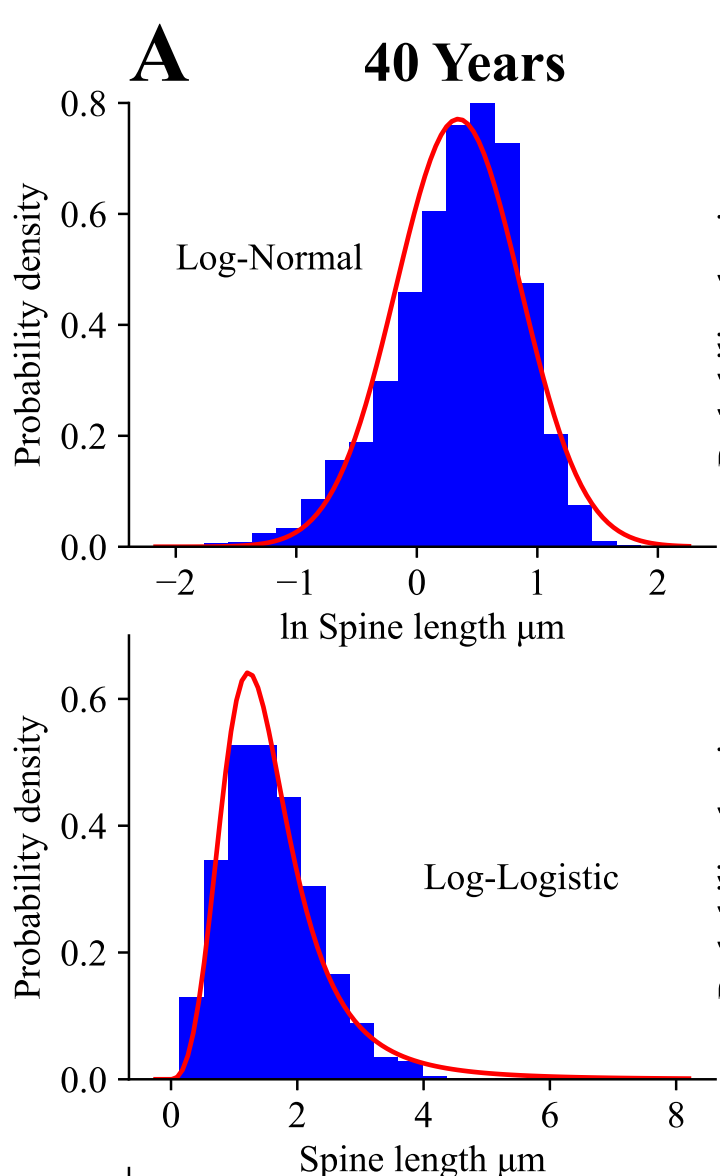

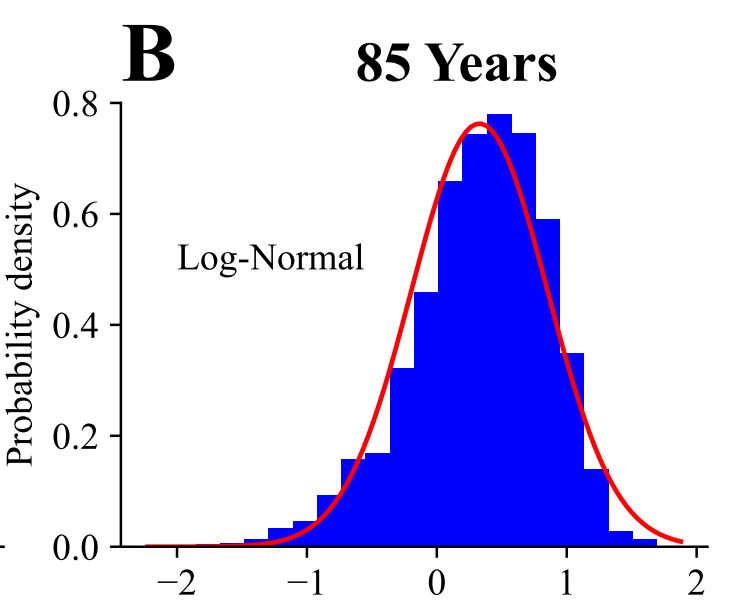

$\ln$ Spine length $\mu \mathrm{m}$

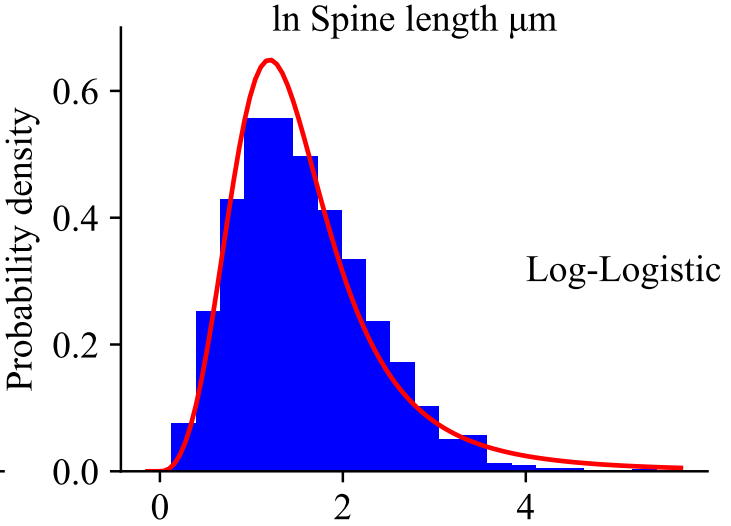

Spine length $\mu \mathrm{m}$

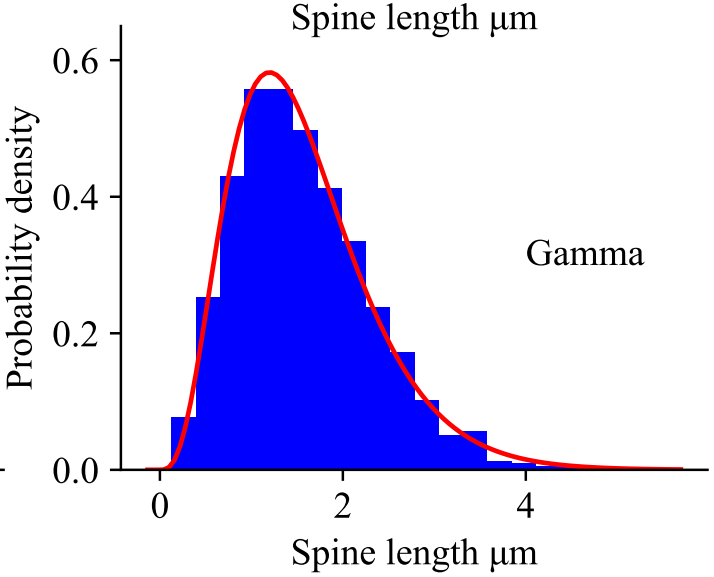

Figure 2 


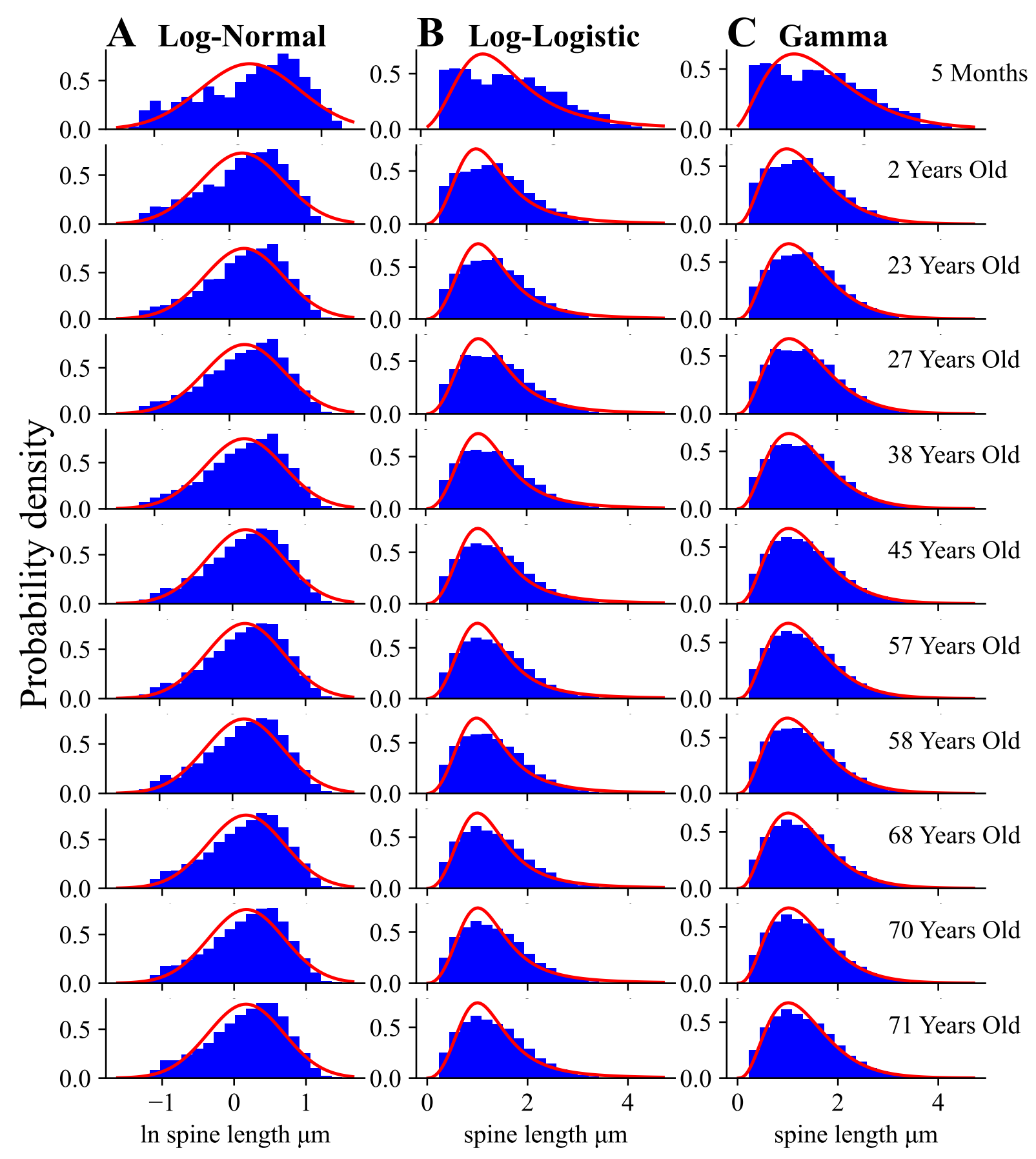

Figure 3 


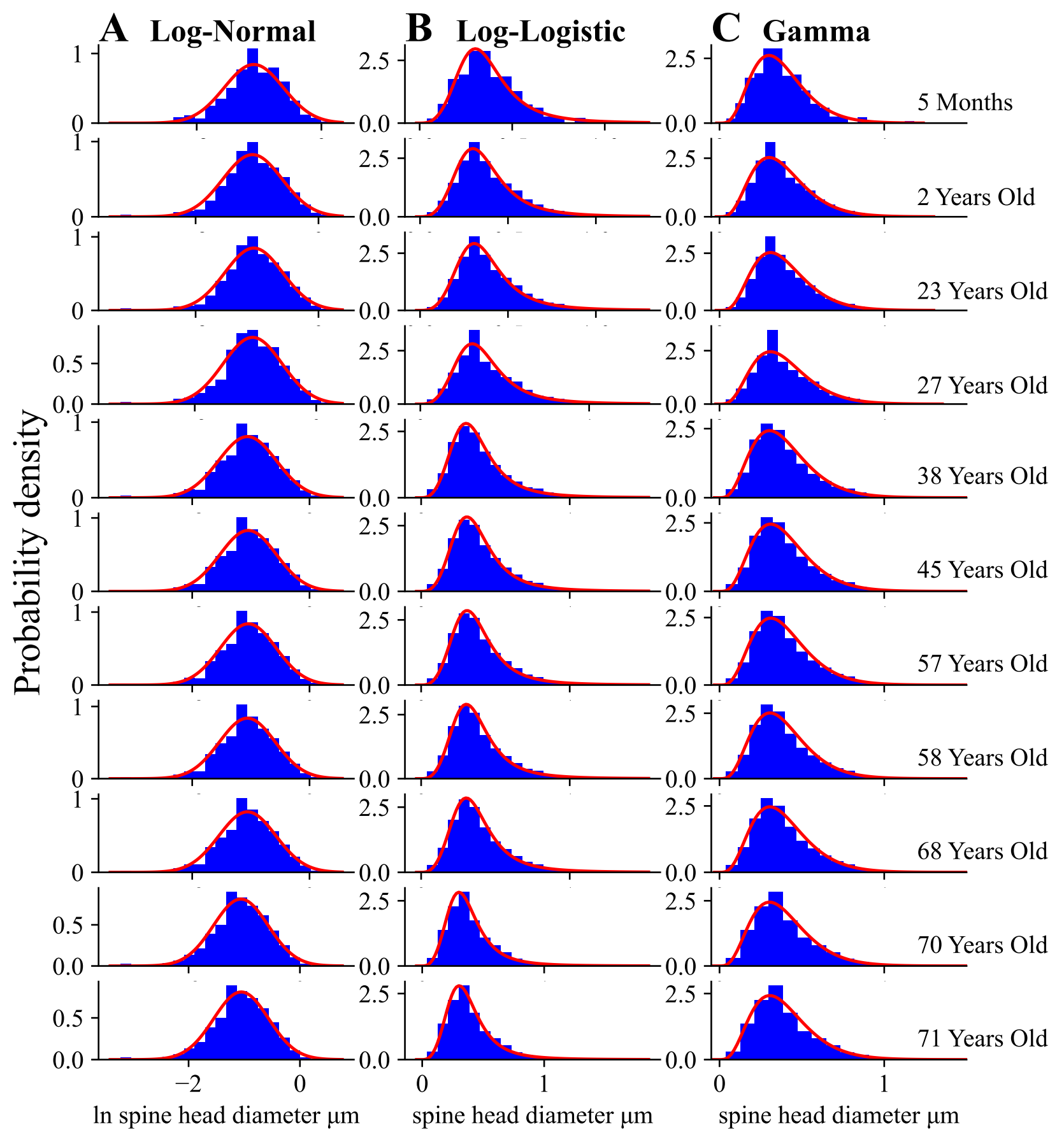

Figure 4 

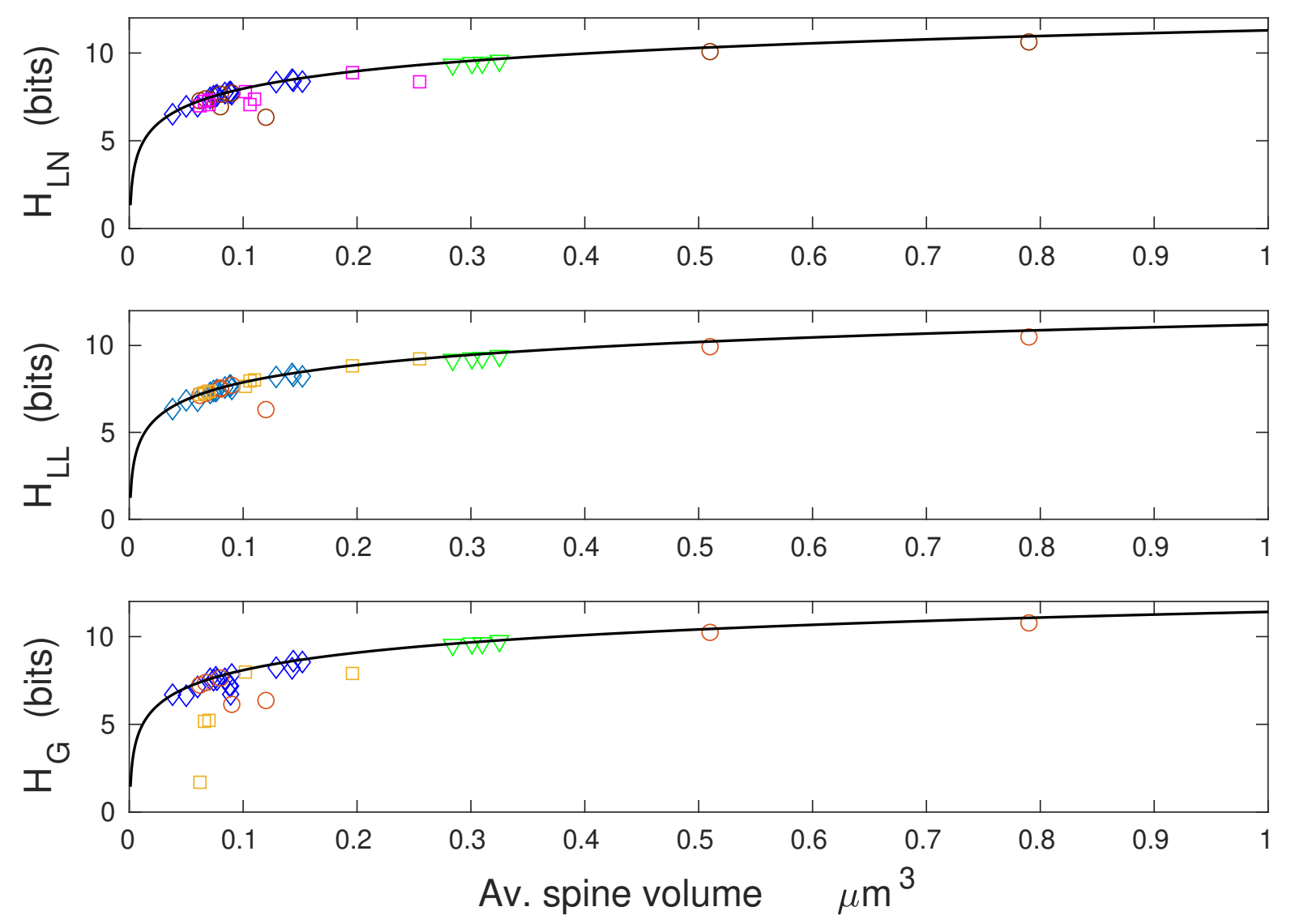

Figure 5 
bioRxiv preprint doi: https://doi.org/10.1101/2021.12.30.474505; this version posted December 30, 2021. The copyright holder for this preprint (which was not certified by peer review) is the author/funder, who has granted bioRxiv a license to display the preprint in perpetuity. It is made available under aCC-BY-NC-ND 4.0 International license.
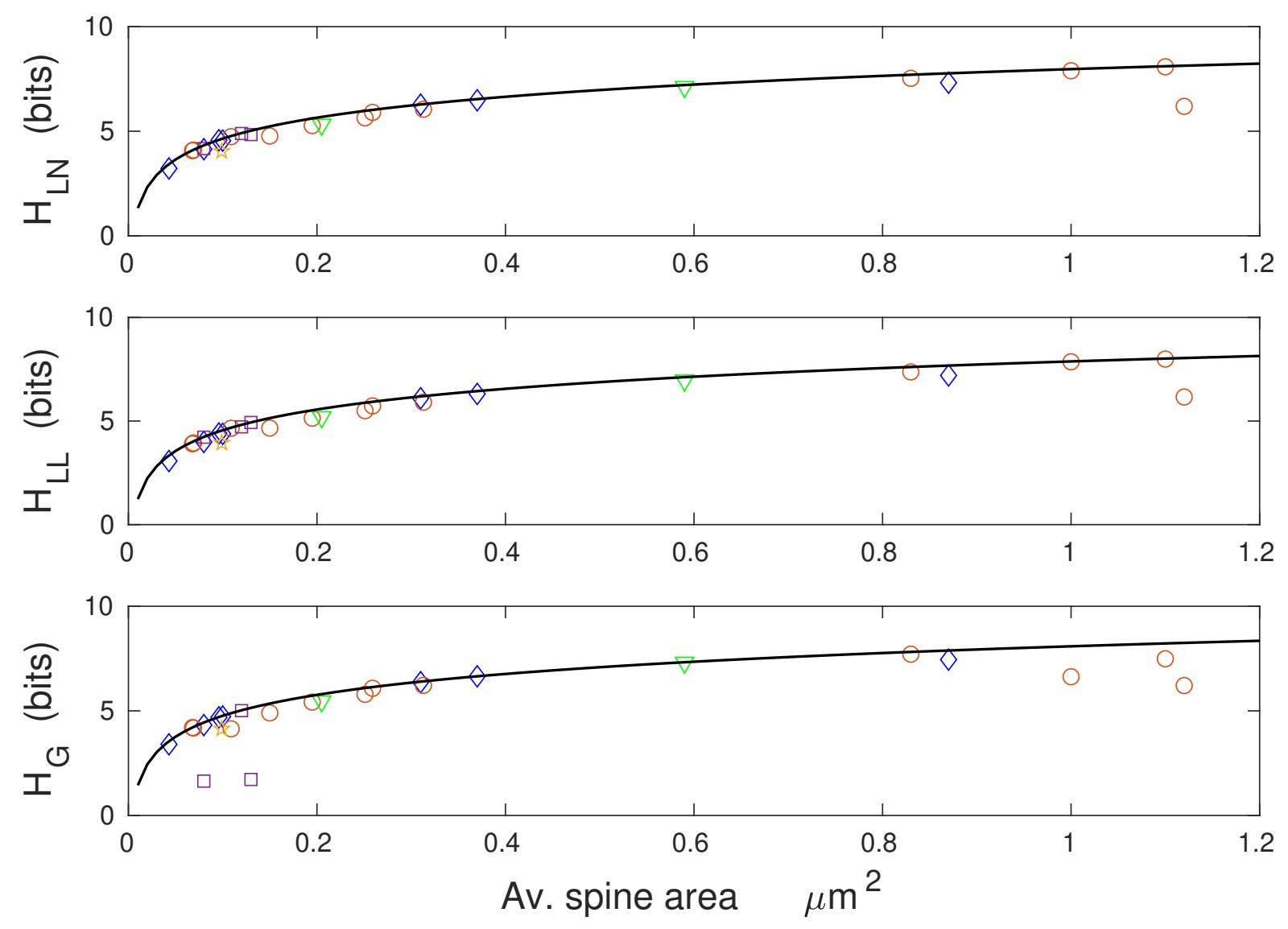

Figure 6 

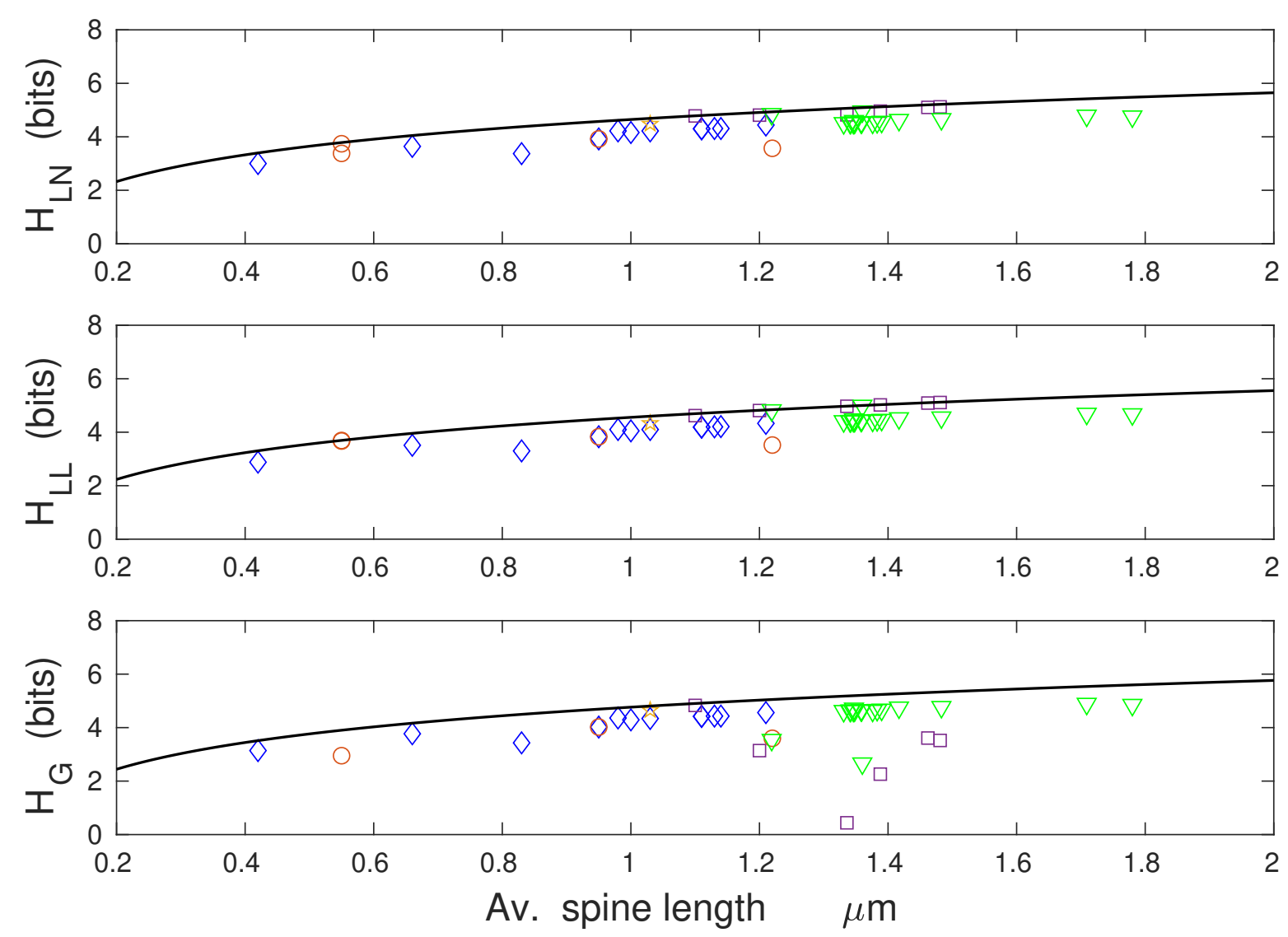

Figure 7 

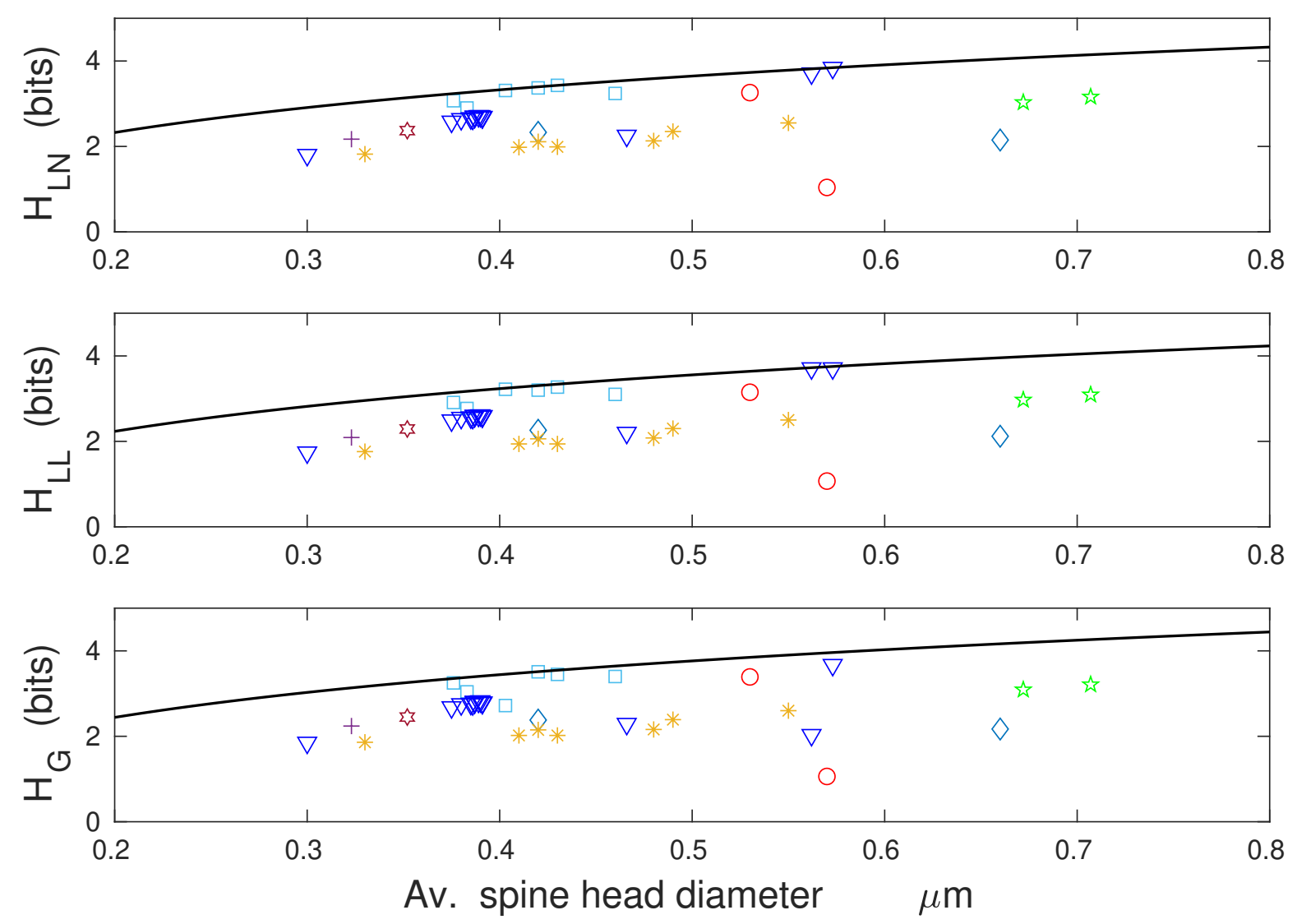

Figure 8 
A

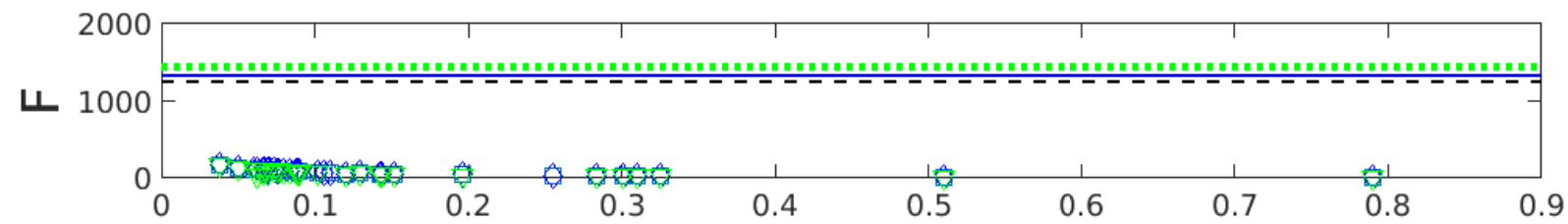

B

Av. spine volume $\left(\mu \mathrm{m}^{3}\right)$

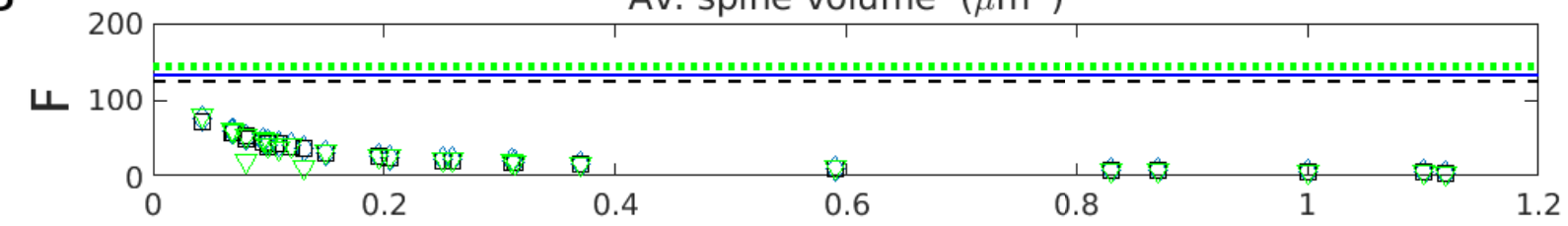

C

Av. spine area $\left(\mu \mathrm{m}^{2}\right)$

ᄂ 10

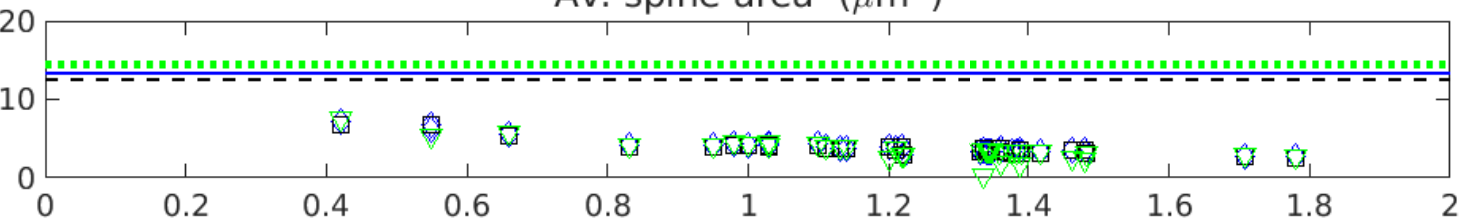

D

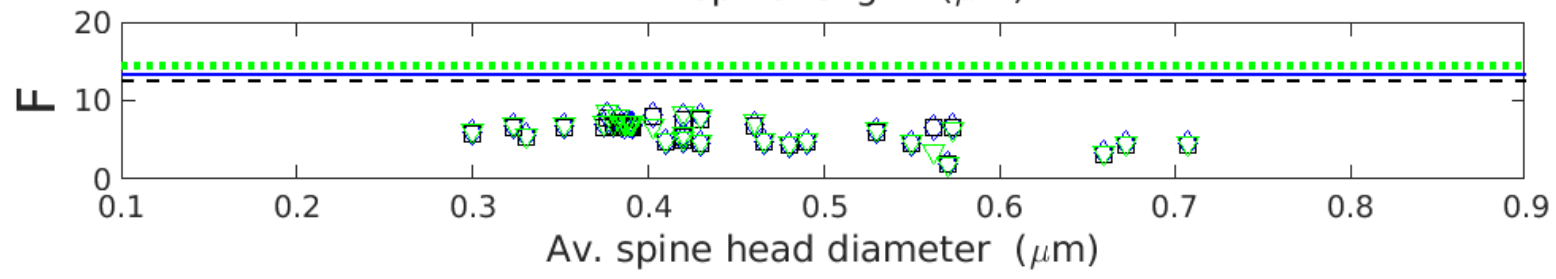

Figure 9 